\title{
Auf den Spuren eines Politikfeldes: Die Institutionalisierung von Internetpolitik in der Ministerialverwaltung
}

\author{
Maximilian Hösl • Ronja Kniep
}

Online publiziert: 5. Februar 2020

(C) Der/die Autor(en) 2020

Zusammenfassung Neue Politikfelder entstehen auch in alten Institutionen. Der Beitrag zeichnet am Beispiel der Internetpolitik in Deutschland die Institutionalisierung eines Politikfeldes nach. Auf der Grundlage einer Kombination von soziologischer Feldtheorie und diskursivem Institutionalismus und gestützt auf Organigramm-Historien, Interviews mit Ministerialbeamten und Behördenpublikationen nimmt er die Entstehung von internetpolitischen Abteilungen in zwei Ministerien in den Blick: dem Wirtschaftsministerium und dem Innenministerium. Der Aufbau von Abteilungen für Internetpolitik ist zugleich eine Form von Diskursinstitutionalisierung und eine Positionierung der Ministerien im Politikfeld, die sich um die Etablierung und Auslegung neuer gemeinwohlrelevanter Schutzgüter dreht. Neben der Wirtschaft und der nationalen Sicherheit tritt nun auch das Internet als Schutzgut hervor, das von den Ministerien im Kontext der bereits bestehenden Schutzgüter semantisch unterschiedlich, jedoch aufbauorganisatorisch ähnlich ausgelegt und als partiell autonomes Politikfeld institutionalisiert wird.

Schlüsselwörter Politikfeld · Feldtheorie · Internet · Institutionalisierung · Ministerialverwaltung · Digitalisierung

Zusatzmaterial online Zusätzliche Informationen sind in der Online-Version dieses Artikels (https:// doi.org/10.1007/s11609-020-00397-4) enthalten.

M. Hösl $(\bowtie) \cdot$ R. Kniep

Wissenschaftszentrum Berlin für Sozialforschung, Reichpietschufer 50, 10785 Berlin, Deutschland

E-Mail: maximilian.hoesl@wzb.eu

R. Kniep

E-Mail: ronja.kniep@wzb.eu 


\title{
On the trails of a policy field: The institutionalization of Internet policy within German ministries
}

\begin{abstract}
New policy fields emerge within old institutions, too. The article shows how this happens by retracing the institutionalization of Internet policy in Germany. It analyzes organizational charts, administrative publications, and biographical interviews with ministerial staff in two ministries - the Ministry of Economy and the Ministry of the Interior - through the lenses of field theory and discursive institutionalism. From this perspective, the formation of new organizational units is to be understood both as an institutionalization of discourse and as a result of ministries strategically positioning themselves in policy fields. At the center of this process is the question what qualifies as a common good that must be protected in the public interest and how. Besides the economy and national security, the Internet emerges as a good to be protected, which, though being interpreted differently by the respective ministries, is institutionalized in similar organizational structures. In both ministries, Internet policy has been institutionalized as a relatively, even though weakly autonomous policy field.
\end{abstract}

Keywords Policy field · Field theory · Internet · Institutionalization · Public administration · Digitalization

\section{Sur les traces d'un champ politique : l'institutionnalisation de la politique numérique au sein de l'administration ministérielle}

Résumé De nouveaux champs politiques voient également le jour dans de vieilles institutions. Cet article retrace l'institutionnalisation d'un champ politique à partir de l'exemple de la politique numérique en Allemagne. Croisant la théorie des champs avec l'institutionnalisme discursif, il se penche sur la création de directions de la politique numérique dans deux ministères, le ministère de l'économie et le ministère de l'intérieur, en s'appuyant sur l'historique d'organigrammes, des entretiens avec des fonctionnaires ministériels et des publications d'administrations. La mise en place de directions consacrées à la politique numérique constitue à la fois une forme d'institutionnalisation du discours et un positionnement des ministères dans le champ politique axés sur l'établissement et l'interprétation de nouveaux biens à protéger d'intérêt public. Outre l'économie et la sécurité nationale, Internet émerge désormais également comme un bien à protéger que les ministères interprètent différemment sur le plan sémantique dans le contexte des autres biens à protéger, quoique de manière similaire sur le plan organisationnel, et qui fait l'objet d'une institutionnalisation en tant que champ politique partiellement autonome.

Mots-clés Champ politique · Théorie des champs · Internet · Institutionnalisation · Administration ministérielle $\cdot$ Numérisation 


\section{Einleitung}

Bereits seit Mitte der 1990er-Jahre ist das Internet in Deutschland ein Bezugspunkt politischer Debatten. Dazu gehören Kontroversen zur Regulierung von Plattformen und ihren Inhalten, zur Verantwortung von Internet-Providern und zur Internetüberwachung. Mit dem Internet haben sich Kommunikations- und Informationspraktiken etabliert, die die Regeln und Begriffswelten bereits bestehender Politikfelder wie Sicherheits-, Wirtschaftspolitik oder Urheberrecht herausfordern. Gleichzeitig haben sich internetspezifische Akteure, Institutionen, Wissensformen und Regelwerke herausgebildet, die auf die Entstehung eines eigenen Politikfeldes hindeuten: dazu gehören Gesetze (Geczy-Sparwasser 2003), Verbände (Lang 2007), eine Netzbewegung (Ganz 2018; Wendelin und Löblich 2013) sowie Fachkompetenzen in den Parteien (Niedermayer 2013), im Parlament (Schwanholz 2019) und in den Ministerien (Pohle et al 2016). Gemeinsam ist den involvierten Akteuren, eine gewisse Internet-Expertise für sich in Anspruch zu nehmen und sich gegen eine rein sicherheits- oder wirtschaftspolitische Lesart des Internets und dessen Regulierung zu positionieren. Zum Teil benennen diese Akteure das neue Politikfeld explizit, wenn auch jeweils unterschiedlich als Netz- oder als Digitalpolitik. Der vorliegende Beitrag macht diese Beobachtung zum Ausgangspunkt einer feldsoziologischen Betrachtung von Internetpolitik ${ }^{1}$. Demnach sind Politikfelder relativ autonome Produktionsräume für eigene Regeln, Denkweisen und Kapitalformen im Sinne einer spezifischen Expertise.

Der Artikel möchte, erstens, einen empirischen Beitrag zur Frage der Institutionalisierung der Internetpolitik leisten, indem er zentrale, aber bisher von der Forschung vernachlässigte Akteure dieser Politik ins Zentrum rückt: die Ministerien und ihre Mitarbeiter. Anhand von Organigramm-Historien und berufsbiografischen Interviews wird die Entstehung neuer organisationaler Strukturen in der Ministerialverwaltung untersucht. Zweitens soll der Artikel konzeptionell dazu beitragen, Politikfelder und ihren Entstehungsprozess besser zu verstehen. Denn obwohl es in Politik und Öffentlichkeit selbstverständlich ist, sachpolitische Bereiche zu unterscheiden, und sich Politikfelder in den Sozialwissenschaften als gängige Analyseeinheiten etabliert haben, gelten ihr Entstehungsprozess sowie ihre theoretische Fundierung weiterhin als Forschungsdesiderat (Döhler 2015; Haunss und Hofmann 2015; Knoke 2004; Loer et al. 2015). Die politische Soziologie hat sich der Entstehung von Politikfeldern bereits feldsoziologisch genähert, jedoch ausschließlich auf transnationaler Ebene (Bernhard 2010; Guiraudon 2003; Mérand 2010; Sending 2015). Der vorliegende Beitrag knüpft an diese feldtheoretische Lesart von Politikfeldern an und überträgt diese auf die nationale Ebene. Zugleich werden Ideen des diskursiven Institutionalismus in den feldsoziologischen Ansatz integriert. Der nachfolgend entwickelte Ansatz stellt Konflikte um Deutungen ins Zentrum und macht sichtbar, wie sich Diskurse in materiellen Strukturen niederschlagen und sich Akteure dadurch relational zueinander im Feld positionieren. Durch diesen Fokus auf die konfligierenden Deutungsmuster von Akteuren reagiert der Beitrag auch auf

\footnotetext{
1 Wir benutzen den wenig gebräuchlichen Begriff „Internetpolitik“, um die stärker politisch konnotierten Begriffe „Netz- und Digitalpolitik“ zu vermeiden.
} 
die Kritik, dass dem soziologischen Neoinstitutionalismus (DiMaggio und Powell 1983; Meyer und Rowan 1977; Scott 2014) eine ,theoretische Mikrofundierung für die Genese und den Wandel von Institutionen“ fehle (Kirchner et al. 2015, S. 198). Drittens bietet der Artikel eine feldsoziologische Auseinandersetzung mit sozio-politischen Transformationsprozessen, die durch das Internet und die Digitalisierung angestoßen werden. Das Internet wird dabei nicht primär als Technologie betrachtet, die in etablierte Felder integriert wird und diese transformiert (vgl. Kropf 2015). Es wird vielmehr selbst zum normativen Bezugspunkt einer neuen Ordnungsbildung in Form eines zunehmend eigenständigen Politikfeldes.

Im folgenden Abschnitt (Abschn. 2) buchstabieren wir das feldsoziologische Verständnis von Politikfeldern aus und definieren die diskursive Institutionalisierung als zentralen Prozess ihrer Entstehung. Aus dieser Sicht bestimmen wir die besondere Rolle von Ministerien in der Politikfeldentstehung. Auf die Erläuterung der Methoden (Abschn. 3) folgt die empirische Analyse, in der wir den Aufbau von internetpolitischen Zuständigkeiten im Wirtschafts- und Innenministerium rekonstruieren (Abschn. 4). Wie die Untersuchung zeigt, ist nicht nur die offene Frage der Schaffung eines eigenen „Digital-“ oder „Internetministeriums“, sondern sind auch der Ausbau eigener Zuständigkeiten innerhalb der etablierten Ministerien Teil der Formierung von Internetpolitik.

\section{Die Institutionalisierung von Politikfeldern in Ministerien}

In der wissenschaftlichen Auseinandersetzung mit Politikfeldern stehen sowohl die theoretisch-konzeptionelle Durchdringung des Phänomens als auch die Reflexion ihrer jeweiligen empirischen Entstehung noch am Anfang. Im Folgenden wird eine feldsoziologische Perspektive auf Politikfelder vorgeschlagen, die ihren Formierungsprozess ins Zentrum rückt. Obwohl für die Etablierung eines neuen Politikfeldes sowohl staatliche als auch nicht-staatliche Akteure relevant sind, konzentriert sich der Beitrag auf Ministerien, die bei der Institutionalisierung nationaler Politikfelder eine zentrale Rolle spielen.

\subsection{Politikfelder als soziale Felder}

Pierre Bourdieus Feldtheorie gilt als ein vielversprechender Ansatz für die Organisationssoziologie, etwa für Analysen interorganisationaler Prozesse (Emirbayer und Johnson 2008). Auch ihr Potenzial für die historische Rekonstruktion der Entstehung von Feldern wird betont (Gorski 2013). Auf Politikfelder und ihre Entstehung wurde seine Feldtheorie bisher jedoch eher selten bezogen, insbesondere nicht auf nationaler Ebene.

Wir begreifen Politikfelder als eine besondere Form sozialer Felder, die das Ergebnis gesellschaftlicher Differenzierung sind. Sie entstehen dadurch, dass sich Akteure aufgrund eines gemeinsamen, aber umstrittenen Anliegens wechselseitig als relevante Akteure wahrnehmen. Feldentstehung bedeutet, dass die Akteure beginnen, ihre (kommunikativen) Handlungen gegenseitig einzubeziehen (Fligstein und Vandebroeck 2014, S. 109) und um Deutungen, Definitionen sowie die Anerkennung einer 
feldspezifischen Expertise zu ringen. Mit anderen Worten, sie kämpfen um symbolische Macht und damit um das feldspezifische symbolische Kapital (Bourdieu 1999, S. 253). Dies gilt insbesondere im Hinblick auf das gemeinsame Anliegen, wie im Fall der hier betrachteten politischen Gestaltung des Internets: angefangen von dessen Infrastruktur- und Anwendungs- bis hin zur Inhaltsebene. Auf der Grundlage des umstrittenen Anliegens verstetigen sich Politikfelder als spezifische und hierarchische Konstellationen von Akteuren, Deutungen, Kontroversen, Institutionen und anerkannter Expertise. Die Politikfeld-Definitionen der Policy-Forschung enthalten ähnliche Kernelemente (vgl. Döhler 2015; Loer et al. 2015; Howlett et al. 2009, S. 81). Die feldtheoretische Rahmung hebt die Relationalität der Akteure und Diskurse sowie die symbolischen und materiellen Konflikte hervor. Eine Feldentstehung im feldsoziologischen Sinne setzt zudem die relative Autonomie dieser Konstellationen voraus. Politikfelder stellen gesellschaftliche Teilbereiche dar, die sich durch eigene Regeln, Bewertungskriterien und einem eigenen Fachwissen von benachbarten Feldern abgrenzen (vgl. Bernhard 2010, S. $77 \mathrm{ff}$.). Der Blick wird dadurch für die Effekte geöffnet, die durch ein Feld selbst produziert werden.

Was aber unterscheidet ein Politikfeld von anderen sozialen Feldern? Erstens geht es in Politikfeldern um unterscheidbare und zum Teil konkurrierende Schutzgüter, die als gemeinwohlrelevant anerkannt sind oder werden sollen. Schutzobjekte der Umweltpolitik wie z. B. Gewässer, Luft und Klima oder der Wirtschaftspolitik wie z. B. Märkte und Wettbewerb müssen Gesellschaften erst einmal als solche entdecken und erfolgreich diskursiv herstellen. Das gelingt aber nur, wenn bestimmte Bezugsobjekte mit einer Allgemeinwohlrelevanz verknüpft werden und diese Relevanz über Fachkreise hinaus anerkannt wird. In Politikfeldern institutionalisiert sich also ein jeweils eigener Schutzanspruch mit Gemeinwohlbezug, der vor der Entstehung des Politikfeldes noch nicht als selbstverständlich galt. Die Diskurse, die auf politikfeldspezifische Schutzgüter Bezug nehmen, sind letztlich „discourses of ultimate value“ (Gorski 2013, S. 334). Unter Bezugnahme auf Max Webers Konzept der Wertsphären sieht Philip S. Gorski in einem solchen Diskurs, der bestimmte soziale Praktiken als inhärent wertvoll und wertvoller als andere definiert, ein zentrales Element der Entstehung neuer Felder (ebd.). Für Politikfelder gilt, dass diese Praktiken bzw. bestimmte Phänomene im politischen Feld und in der medialen Öffentlichkeit als regelungsbedürftige und legitime Streitgegenstände anerkannt werden, kurz: als gemeinwohlrelevant. Gleichzeitig werden diese spezifischen Praktiken und Schutzgüter von bestimmten Akteuren in den Politikfeldern als die wichtigsten und dringlichsten reklamiert.

Die Entstehung eines Politikfeldes „Internet“ setzt voraus, dass dem Internet bzw. den internetbezogenen Praktiken der Status von schützenswerten Gütern zuerkannt wird, und zwar ein Wert, der sich nicht ohne Weiteres bereits existierenden wirtschafts- oder sicherheitspolitischen Vorstellungen unterordnen lässt. Gleichzeitig bleibt die Autonomie des Feldes relativ, weil jedes Feld stets auch heteronomen Einflüssen unterliegt (Bourdieu 1999, S. 349). Das Spannungsverhältnis zwischen Heteronomie und Autonomie äußert sich zwischen und innerhalb von Politikfeldern als Spannungsverhältnis zwischen verschiedenen Schutzgütern und den entsprechenden politischen Zuständigkeiten. Auf dieser Grundlage lässt sich für das internetpolitische Feld ein diskursiver Raum mit zwei Extrempositionen aufspannen: Die 
autonome Position konkretisiert sich im Anspruch von Internetaktivisten, das Internet als „,reien“, „offenen“ und „,globalen“ Kommunikationsraum zu schützen (Ganz 2018; Sell 2017, S. 241). Diese Forderung steht in Verbindung mit Ideen wie Hierarchiefreiheit, Offenheit und neutrale Datenübertragung, die in einer bestimmten historischen Situation in die technische Struktur des Internets eingeschrieben wurden (Clark 2016) und aus dieser Perspektive als ursprünglich, einzigartig und besonders schützenswert wahrgenommen werden. Heteronomere Positionen betonen hingegen die durch das Internet entstehenden Wirtschaftschancen oder die Notwendigkeit der Durchsetzung von Sicherheit im Internet, d.h. sie sehen das Internet eher durch die Brille bereits bestehender Schutzgüter.

Zweitens unterscheiden sich Politikfelder von vielen anderen sozialen Feldern dadurch, dass in ihnen Akteure aus ganz unterschiedlichen sozialen Feldern der Gesellschaft in Aushandlungsprozessen aufeinandertreffen (z. B. Politik, Verwaltung, Wirtschaft und Zivilgesellschaft). Drittens ringen Akteure in Politikfeldern darum, ihren Deutungen durch Institutionalisierung einen möglichst weitreichenden Wirkungs- und Geltungsbereich zu verleihen, etwa in Form von Gesetzen. Dadurch haben Politikfelder einen gesamtgesellschaftlichen Koordinationseffekt, der andere soziale Felder und deren Autonomie beeinflusst. Vincent Dubois (2015, S. 215) bezeichnet daher Politikfelder als Meta-Felder, um ihre übergeordnete Ordnungsleistung zu beschreiben.

\subsection{Politikfelder und ihre diskursive Institutionalisierung}

In Politikfeldern steht symbolische Macht auf dem Spiel. Akteure ringen nicht nur um Deutungen, sondern auch darum, sie auf Dauer zu stellen - sie ringen um deren Institutionalisierung. Um die Institutionalisierung von Politikfeldern in Ministerien nachzuzeichnen, integrieren wir das Konzept der Diskursinstitutionalisierung (Hajer 1995, S. 60 ff.) in den feldtheoretischen Ansatz. ${ }^{2}$ Die Konzeptualisierung des Feldes als subjektiver symbolischer Raum gilt bei Bourdieu als weniger ausgearbeitet als dessen Konzept des sozialen Raums objektiver Beziehungen (Gorski 2013, S. 334). Gorski legt den Akzent auf Diskurse und Narrative, um diesen blinden Fleck auszuleuchten. Maarten A. Hajers Konzept der Diskursinstitutionalisierung, das die Beziehung zwischen Diskursen und ihren verfestigten Formen - den Institutionen - hervorhebt, bietet eine Möglichkeit, die symbolisch-diskursiven Relationen in Feldern stärker zu erfassen.

Wir verstehen einen Diskurs als Ensemble zusammenhängender Ideen, Frames, Definitionen und Narrative, die sozialen und materiellen Phänomenen Bedeutung zuschreiben (Hajer 1995, S. 60 ff.). Zum einen (re)produzieren Akteure im Feld Diskurse und versuchen dabei, bestimmte Sichtweisen als Common Sense zu etablieren. Zum anderen strukturieren Diskurse die Wahrnehmung der Akteure im Feld. Einige Diskurse, jedoch bei weitem nicht alle, schlagen sich in Institutionen nieder (Phillips et al. 2004, S. 638) und tragen somit maßgeblich zur Gestaltung und Etablierung eines Politikfeldes bei. Deshalb liegt der Fokus der Politikfeldanalyse in

2 Zur Beziehung zwischen Feld- und Diskurstheorie siehe Diaz-Bone (2006) und Schmidt-Wellenburg (2013). 
diesem Beitrags nicht auf der Untersuchung der im Feld produzierten oder in das Feld hineinwirkenden Diskurse, sondern auf dem Prozess der diskursiven Institutionalisierung. Die gegenseitige Verstärkung zwischen den Semantiken der Diskurse und Institutionen ist ein wesentliches Merkmal der Entstehung eines Politikfeldes (Bernhard 2011, S. 433).

Die Feldsoziologie hebt die objektivierende Kraft von Institutionen hervor: „Die Objektivierung in Institutionen garantiert den Bestand [...] der materiellen wie symbolischen Errungenschaften, ohne daß die Subjekte sie ständig und vollständig durch bewußtes Handeln wiedererschaffen müßten“ (Bourdieu 1993, S. 239), d.h. die Institutionen werden anerkannt und nicht immer wieder hinterfragt. Den als selbstverständlich begriffenen Institutionen ging eine akteursgetriebene Institutionalisierung voran, die jedoch in Vergessenheit geriet (Bourdieu 1994, S. 3). Die Diskursinstitutionalisierung ist also kein Automatismus, sondern ein Prozess einer graduell zunehmenden, aber grundsätzlich auch umkehrbaren Objektivierung durch Akteure (Berger und Luckmann 1966). Bevor sich die objektive Kraft von Institutionen sowohl in der Breite (z. B. hinsichtlich des Akteursspektrums) als auch in der Tiefe (hinsichtlich des Grades der Verinnerlichung) ausbildet, eignen sich Akteure in lokalen Experimenten bestimmte Denk- und Handlungsmuster an (Habitualisierung) und schaffen organisationale Strukturen, um auf die Problemwahrnehmung bestimmter Diskurse zu reagieren (Tolbert und Zucker 1999, S. 180). Die Entstehung von Politikfeldern kann als eine beginnende Institutionalisierung verstanden werden, die zunächst eine minimale Tiefe und Breite aufweist.

Die Empirie dieses Beitrags widmet sich lediglich einem Ausschnitt der Entstehung des internetpolitischen Feldes, weshalb eine Beobachtung des Institutionalisierungsprozesses in seiner ganzen Breite und Tiefe nicht möglich ist. Der Fokus liegt darauf, wie als Effekt bestimmter Diskurse organisationale Strukturen entstehen und wie sie von den involvierten Akteuren argumentativ legitimiert werden. Der Effekt von Diskursen und ihrer Institutionalisierung manifestiert sich in der Wechselwirkung von Vorstellungen ,,in den Köpfen“ der Akteure mit der materiellen Welt: „We organize our material world in accordance with our mental categories, and the two become self-reinforcing“ (Scott 2014, S. 149). Wenn Materialisierungen (formale Prozesse, Dokumente, Technologien) und kognitive Kategorien sich wechselseitig verstärken, dann sind sie nicht eine schlichte Repräsentation einer Realität außerhalb ihrer selbst, sondern sie tun etwas. Diesen performativen Charakter von Materialisierungen erfasst die Akteur-Netzwerk-Theorie mit dem Begriff der Einschreibung. Einschreibungen in Form von Codes, Texten oder Gesetzen sind ,mobilization[s] of the world" (Latour 1999, S. 99), die zu sozialer Ordnung beitragen oder die bestehende Ordnung unterminieren. Als Einschreibungen werden Diskurse in unterschiedlichen Ausprägungen auf Dauer gestellt, transportabel gemacht, dabei aber auch transformiert (Dölemeyer und Rodatz 2010, S. 6). Eine Einschreibung führt dazu, dass andere mögliche Seinszusammenhänge unsichtbar werden (Nimmo 2011, S. 114). Ein entscheidender Prozess der Politikfeldentstehung ist demnach die Objektivierung mentaler Kategorien als Bestandteile von feldspezifischen Diskursen in materiellen Strukturen. Dazu zählen auch Einschreibungen in Form von Organisationseinheiten wie Abteilungen oder Referaten. 
Objektivierung und Materialisierung sind keine linearen und irgendwann abgeschlossenen Prozesse, sondern können erneut zum Gegenstand von Deutungskämpfen werden und so zu einer De- und Re-Institutionalisierung führen. Das gilt vor allem für den Beginn der Politikfeldentstehung, wenn etablierte Materialitäten und objektivierte Ideen infrage gestellt werden. Akteure tragen in ihren Auseinandersetzungen um die Diskursinstitutionalisierung zu einem Set an feldspezifischen Einschreibungen bei, die wiederum feldspezifische Konflikte verstetigen. Auf diese Weise bildet sich eine relative Autonomie eines Politikfeldes mit bestimmten Handlungslogiken heraus, die auf das Feld und seine Akteure zurückwirken und es von anderen Feldern unterscheidbar macht. In diesem Sinne sind Politikfelder relativ autonome, konflikthafte Produktionsräume für Bedeutungen und daraus abgeleitete Handlungsalternativen, Kompetenzen und Institutionen.

\subsection{Ministerien in Politikfeldern}

Ministerien stehen in einem Spannungsverhältnis von multiplen Feldern wie Politik, Recht, Wirtschaft und von bestimmten Politikfeldern. Ihre Binnenstruktur ist zwar von den Eigenheiten der Ministerialverwaltung abhängig, z.B. vom Hierarchieprinzip und vom Prinzip der Zuständigkeiten, d.h. der Organisation nach „rational abgegrenzten sachlichen“ Aufgabenbereichen (Weber 1980, S. 125). Aber auch die multiplen Umwelt- bzw. Feldbezüge beeinflussen die Aufbauorganisation (Lichtmannegger 2018; Richter 2012, S. 95). Der vorliegende Beitrag nimmt die Binnenstruktur von Ministerien in Korrespondenz mit einem entstehenden Politikfeld in den Blick, richtet also den Fokus auf die politikfeldspezifischen Logiken, die ein Ministerium prägen bzw. durch Ministerien selbst erst geprägt werden. Zu diesen Politikfeldlogiken zählen fachliche Expertise, Problemwahrnehmungen und bestimmte politische Handlungsoptionen. Thurid Hustedt (2013, S. 130) spricht mit Blick auf diese fachlich-inhaltliche Handlungsorientierung in Ministerien vom „Denken in Policies“ als „kognitive Schablone“. Das gilt nicht nur für ganze Ministerien. Auch Abteilungen oder Referate sind entsprechend ihrer Zuständigkeitsbereiche von eigenen Denkschemata geprägt, zeichnen sich also durch ein „Denken vom ,eigenen' Referat her" aus (ebd., S. 131). Zudem sind Ministerien häufig nicht nur für ein Politikfeld zuständig. Das Bundesministerium für Wirtschaft und Energie hat etwa Zuständigkeiten sowohl für die Industrie- als auch die Energiepolitik.

Mit einem neuen Politikfeld entsteht also nicht zwangsläufig ein neues Ministerium. Im gesellschaftlichen und diskursiven Entdeckungsprozess neuer Themen- und potenzieller Politikfelder beginnen zunächst etablierte Ministerien, sich in Bezug auf diese Diskurse und relational zu den anderen Akteuren zu positionieren. Aus feldtheoretischer Sicht konzentrieren Ministerien hierbei Ressourcen und stärken damit ihre Position, um Deutungsmacht ausüben zu können (Bourdieu 1994, S. 4). Die Positionierung von Ministerien, etwa durch den Aufbau von Expertise, Zuständigkeiten und Organisationseinheiten, kann als Prozess diskursiver Institutionalisierung von Politikfeldern verstanden werden. Ministerien nehmen in diesem Prozess zwei miteinander verschränkte Rollen ein: 
Erstens sind sie durch die Wahrnehmung ihrer Kernaufgabe, der Politikformulierung (Derlien 1995, S. 80), Akteure im Feld und Ko-Produzenten von Politikfeldern. Sie sind Teil des Ringens um Diskursinstitutionalisierung, denn sie ko-strukturieren Diskurse, indem sie das Vokabular, die Problemwahrnehmungen, Konflikte und Handlungsoptionen im Politikfeld prägen. Das geschieht zum Beispiel, wenn Minister an die (Fach-)Öffentlichkeit treten oder Abteilungen an Strategiepapieren und Gesetzesentwürfen arbeiten. In entstehenden Politikfeldern müssen Ministerien zunächst Zuständigkeiten und relevante Expertise aufbauen, um als anerkannte Akteure in diesen Politikfeldern auftreten zu können, aber auch, um im jeweiligen Feld als interventions- und koordinationsfähig zu gelten (Richter 2012, S. 97). Nicht nur zwischen den Ministerien, auch ministeriumsintern findet ein Ringen um Expertise und Zuständigkeiten statt (vgl. Emirbayer und Johnson 2008, S. 22).

Zweitens sind Ministerien Adressaten der Diskursinstitutionalisierung für die verschiedenen Akteure in den Politikfeldern und reflektieren damit die jeweilige Politikfeldentstehung. Die diskursiven Verhältnisse im Politikfeld prägen die Definitionen dessen, was als rational abgrenzbare Zuständigkeit gilt, und somit den Zuschnitt einzelner Abteilungen und Referate. Auch hierbei sind Ministerien nicht (nur) als Einheit zu denken: Bestimmte Abteilungen sind in je eigene Diskurse mit einer Fach-Community (Behörden, Wissenschaft, Unternehmen) involviert. Die Ministerien eignen sich mitunter als institutionelle Ziele für Akteure, die ihre Deutungen auf Dauer stellen möchten, etwa durch Einflussnahme auf Gesetzesentwürfe. Die Institutionalisierung findet dabei stets gefiltert durch die internen Deutungskämpfe im jeweiligen Ministerium statt.

Ministerien sind also aktive Ko-Produzenten von Politikfeldern und ihren Handlungslogiken. Gleichzeitig sind sie durch ihre Adressaten-Rolle eine Art Resonanzkörper für entstehende Politikfelder. Aufgrund dieser beiden verschränkten Rollen lassen sich dort politikfeldspezifische Denkschemata, Narrative, Deutungen und Konflikte besonders gut beobachten - zumindest solche, die staatlich akzeptiert und dominant sind oder waren. In den folgenden Abschnitten wird am Beispiel der Internetpolitik der Aufbau von Expertise in Form von Organisationseinheiten als Diskursinstitutionalisierung rekonstruiert.

\section{Methodische Herangehensweise}

Zunächst wollen wir jedoch unsere methodische Herangehensweise darlegen, d.h. die methodologische Verortung des Beitrages, den Zyklus des Forschungsprozesses sowie die Begründung der Fallauswahl. Zudem werden die Organigramm-Analyse als Methode und die Organigramme als spezifischer Datentyp genauer beschrieben.

\subsection{Methodologische Verortung, Forschungszyklus und Fallauswahl}

Die Internetpolitik eignet sich gerade deshalb für die Untersuchung einer Politikfeldentstehung, weil die Eigenständigkeit des Feldes und dessen Beschaffenheit im Vergleich zu den etablierten Politikfeldern bis heute umstritten geblieben ist. Das zeigt sich in den unterschiedlichen Begriffen (Netz- oder Digitalpolitik), aber auch in 
der umstrittenen Frage: „Brauchen wir ein Digitalministerium?“ Wir sind der Frage nachgegangen, welche internetbezogenen Diskurse und Konflikte sich wie innerhalb der ministeriellen Aufbauorganisation institutionalisiert haben. Indem wir die Herausbildung von Organisationseinheiten im Zeitverlauf nachvollziehen, betreiben wir eine ,,archaeological deconstruction“ (Fligstein und Vandebroeck 2014, S. 122; mit Berufung auf Bourdieu 1994) des internetpolitischen Feldes. Da wir Politikfelder als sozio-politisch konstruierte Räume betrachten, verfolgen wir einen interpretativen Ansatz. Der Fokus liegt auf der Sinnbildung in historisch kontingenten Situationen und auf einer ,constitutive causality“ (Schwartz-Shea und Yanow 2012, S. $51 \mathrm{ff}$.), also auf einer Ausweitung oder Einschränkung als sinnvoll erscheinender Folgehandlungen und -interpretationen durch die Deutungen der Akteure.

Die empirische Analyse stützt sich auf eine Triangulation von Organigramm-Historien, von narrativen berufsbiografischen Interviews mit leitenden Ministerialbeamten $^{3}$ sowie von Behörden- und Medienpublikationen. Organigramme sind, wie Texte oder Gesetze, Einschreibungen von Diskursen. Organigramm-Historien sind Darstellungen chronologischer Entwicklungen von Organisationseinheiten, z. B. Abteilungen (vgl. die Online-Dokumente 1 und 3). ${ }^{4}$ Der Untersuchungszeitraum beginnt mit den ersten in den Organigrammen sichtbaren IT- und internetrelevanten Kompetenzen 1995 und endet im Jahr 2016.

Die interpretative Analytik folgt einem zyklischen Forschungsprozess, in dem die unterschiedlichen Datentypen Schritt für Schritt aufeinander bezogen wurden, um den Aufbau der internetpolitischen Kompetenzen in zwei Ministerien nachzuzeichnen (vgl. zum ,fitting-Prozess“ Diaz-Bone 2006, S. 79; Schmidt-Wellenburg 2013, S. 464). Die Ergebnisse der Organigramm-Analyse informierten die Interviewführung. Das aus der Analyse der formalen Veränderung der Organigramme hervorgegangene Vorverständnis des Aufbauprozesses wurde durch Deutungen des Wandels durch die Interviewpartner angereichert und korrigiert (ebd., S. 468). Sowohl die Organigramme als auch die Interviews verweisen jeweils auf Kontexte und Hintergründe, die mit zusätzlichen Publikationen weiter erschlossen wurden. Das Verständnis des Aufbauprozesses wurde dadurch entweder ergänzt oder herausgefordert (zum Zyklus des Forschungsprozesses vgl. Online-Tabelle 1). Dieses Forschungsdesign ermöglichte es, die Relationen zwischen den Deutungs- und Abwägungsprozessen innerhalb und außerhalb der Ministerien sowie die Veränderungen in der Aufbauorganisation zu analysieren und im Zuge des Forschungszyklus zu einer empirisch gestützten Erzählung zu verdichten.

\footnotetext{
3 Im Zeitraum von April 2015 bis September 2016 wurden insgesamt acht Interviews mit leitenden Ministerialbeamten geführt, die langjährig den in den Organigrammen sichtbaren Wandel mitgestaltet haben. Bei den Berufsbiografien handelt es sich weitgehend um Karrieren innerhalb der untersuchten Ministerien. Die Interviews dauerten zwei bis zweieinhalb Stunden. Im Folgenden werden den Äußerungen der einzelnen Interviewpartner zwecks Anonymisierung Nummern zugeordnet („I1“, „I2“ etc.).

4 Die Online-Dokumente sind als Zusatzmaterial auf der Webseite des Artikels verfügbar. Die Daten und Organigramme wurden anhand folgender Quellen zusammengestellt: Bund transparent. Parlament, Regierung, Bundesbehörden. Organisation, Gremien, Anschriften, Namen. Ausgaben 1995 bis 2014; Staatshandbuch Bund. Verzeichnis der Behörden mit Aufgabenbeschreibungen und Adressen. Ausgaben 1995 bis 2015 .
} 
Wir betrachten zwei Fälle: die Unterabteilung „Nationale und europäische Digitale Agenda"5 im Bundesministerium für Wirtschaft (BMWi) und die „Abteilung IT"6 im Bundesministerium des Innern (BMI). Von den drei federführenden Ministerien der Digitalen Agenda 2014-2017 haben das BMWi und das BMI relativ früh internetpolitische Kompetenzen aufgebaut, während das Bundesministerium für Verkehr und digitale Infrastruktur (BMVI) erst ab 2015 vermehrt einschlägige Zuständigkeiten erhielt. Auch gegenüber der gesamten Ministerialverwaltung zeichnen sich das BMI und das BMWi durch den quantitativ höchsten Anteil an internetpolitischen Zuständigkeiten aus (Hösl et al. 2019).

Drei Zeitpunkte sind für die Analyse besonders relevant: 1995 im BMWi, 2002 im BMI sowie 2014 in beiden Ministerien. Sie markieren den Beginn umfassenderer Veränderungen in den Organigramm-Historien. Der zeitliche Vergleich ermöglicht Interpretationen im Hinblick auf die Institutionalisierung von Internetpolitik in den Ministerien.

\subsection{Organigramm-Analyse}

Organigramme der Ministerien stellten den Ausgangspunkt des Forschungszyklus dar. Daher bedarf ihre Interpretation im Lichte unseres theoretischen Ansatzes einer gesonderten Aufmerksamkeit. Einschreibungen von Diskursen werden in Organigrammen auf semantischer und aufbauorganisatorischer Ebene beobachtbar.

Auf semantischer Ebene manifestieren sich Einschreibungen durch die Namensgebung von Organisationseinheiten und deren Aufgabenbeschreibung. Auch weil Ministerien relativ stabile institutionelle Gebilde sind, verweisen neue Einschreibungen auf eine gewisse Dominanz bzw. symbolische Macht zugehöriger Diskurse. Namensgebungen reflektieren jedoch nicht nur dominante Diskurse. Denominationen wirken performativ, indem sie nach innen in die Organisation, aber auch nach außen in die Gesellschaft sowie gegenüber Fach-Communities signalisieren: Wir sind zuständig (I2 2015; I1 2015; Fleischer et al. 2018). Ministerien betreiben mit der Benennung Definitionsarbeit am umstrittenen Anliegen und an den Schutzgütern und positionieren sich in der Frage: Um was geht es, was steht auf dem Spiel? Schließlich erfolgt auch eine Anschlusskommunikation an Begriffe, die mitunter in programmatischer Absicht gewählt werden. Referate bevorzugen aufgrund der Signalfunktion nach außen häufig politisch ansprechende Namen, während die Zentralverwaltung auf eine logische, möglichst überschneidungsfreie Einpassung in die Ministerialstruktur abzielt (I2 2015; I1 2015). Der Einschreibungsprozess wird also auf der semantischen Ebene auch durch verwaltungsinterne Überlegungen geprägt, nicht nur von Kategorien dominanter politikfeldspezifischer Diskurse. Trotzdem lässt die Korrespondenz der Namensgebung von Referaten oder Abteilungen mit den Kategorien, Definitionen und Begriffen aus bestimmten Diskursen Rückschlüsse auf

\footnotetext{
5 So heißt die Unterabteilung seit 2016. 2015 hieß sie noch „Digitale Agenda, Digitale Medien“. Deshalb taucht sie unter diesem Namen in der Organigramm-Historie in Online-Dokument 1 auf.

6 Seit 2018 heißt sie ,Abteilung Digitale Gesellschaft, Verwaltungsdigitalisierung und Informationstechnik (DG)“ bzw. „Abteilung DG“.
} 
die zu diesem Zeitpunkt dominanten, für die Institutionalisierung ausschlaggebenden Diskurse zu.

Auf aufbauorganisatorischer Ebene lässt sich die Herausbildung verschiedener organisationaler Strukturen beobachten. Die organisationalen Strukturen stehen auch für die Positionierung in Politikfeldern durch den Aufbau personeller und finanzieller Ressourcen sowie von Expertise. Zunächst lässt sich unterscheiden, ob Einheiten außerhalb oder innerhalb der ordentlichen Hierarchie angesiedelt sind. Organisationseinheiten innerhalb der Linienorganisation zeichnen sich in der Regel durch eine höhere Sichtbarkeit und Permanenz aus als temporäre Einheiten außerhalb der ordentlichen Hierarchie. Der Institutionalisierungsgrad nimmt in Tiefe und Breite zu ausgehend von Projektgruppen oder Stäben, die noch außerhalb der ordentlichen Hierarchie stehen, über Referate bis hin zu Abteilungen innerhalb der ordentlichen Hierarchie (vgl. Online-Tabelle 2). Eine Politikfeldentstehung drückt sich demnach in Ministerien durch die Konzentration von Ressourcen in Verbindung mit der Einschreibung von Diskursen in Organisationseinheiten aus.

\section{Auf dem Weg zur Internetpolitik: Diskursinstitutionalisierung in Ministerien}

Die folgende Analyse nimmt sich jeweils der Entstehungsepisoden internetpolitischer Zuständigkeiten im BMWi 1995 und im BMI 2002 an und untersucht im Anschluss die umfassende Neuordnung der Zuständigkeiten in beiden Ministerien ab 2014. Der Beschreibung der organisationalen Strukturen wird jeweils eine kurze Analyse der relevanten Diskurse vorangestellt. Auf diese Weise zeichnen wir die diskursive Institutionalisierung nach und interpretieren dann die Beobachtungen aus politikfeldanalytischer Perspektive. Dazu gehört, konkurrierende Deutungen und Schutzgüter im Spannungsfeld von Heteronomie und Autonomie herauszuarbeiten.

\subsection{Bundesministerium für Wirtschaft: Von der Informationsgesellschaft zur Digitalisierung}

Im BMWi haben zwei Diskurse die Institutionalisierung von Internetpolitik geprägt. Im Kontext des Diskurses zur Informationsgesellschaft der 1990er-Jahre baute das Ministerium erste internetpolitische Zuständigkeiten auf. 2015 kamen schließlich neue Kompetenzen vor dem Hintergrund des Diskurses zur Digitalisierung und der Digitalen Agenda hinzu. Eine eigene Abteilung für Internetpolitik entstand.

\subsubsection{Beginnende Institutionalisierung: Der Weg in die Informationsgesellschaft (1995-1999)}

Die Idee der Informationsgesellschaft geht auf akademische Debatten seit den 1970er-Jahren zurück und wurde in den 1990er-Jahren zu einem Diskurs mit hoher politischer Relevanz. Internationale und europäische Gremien sowie Regierungen griffen ihn auf, etwa im Bericht einer EU-Expertengruppe (vgl. Bangemann-Report European Commission 1994), einer G7-Konferenz (1995) sowie in einer Reihe von 
Policy-Initiativen. Kern des Diskurses war die Annahme eines Wandels von der Industrie- zur Informationsgesellschaft. Die Gremien und Regierungen verbanden mit diesem Wandel eine optimistische Erwartung - insbesondere hinsichtlich des wirtschaftlichen Wachstums und der gesellschaftlichen Entwicklung (Audenhove et al. 2003, S. 82). Um dieses Potenzial auszuschöpfen, das den neuen Informationsund Kommunikationstechnologien (IKT) zugeschrieben wurde, sollten soziale und organisatorische Hürden überwunden werden (Jørgensen 2012). In Deutschland bildete der Diskurs um die Informationsgesellschaft in den 1990er-Jahren den Bezugsrahmen, unter dem das Internet erstmals im politischen Feld diskutiert und strategisch adressiert wurde (vgl. BT-Drs. 13/4000).

Der Diskurs zur Informationsgesellschaft wurde 1995 im BMWi in einer Arbeitsgruppe eingeschrieben. In der Abteilung „Gewerbliche Wirtschaft, Industriepolitik“ im BMWi war bereits vor 1995 ein Referat mit Verantwortlichkeiten für die IKT angesiedelt. Aus dieser Abteilung ging 1995 die Idee zur Einrichtung der Arbeitsgruppe „Informationsgesellschaft“ hervor (I3 2016). Die Arbeitsgruppe war ein lokales Experiment außerhalb der ordentlichen Hierarchie. Die Kompetenzen der Gruppe wurden 1996 in die ordentliche Hierarchie überführt und verstetigt. 1999 wurde aus diesen Zuständigkeiten die Unterabteilung „Informationsgesellschaft, Medienrecht“, die nun außerhalb der Industrieabteilung ausschließlich für Belange der Informationsgesellschaft zuständig war (vgl. Online-Dokument 1). Hier deutet sich die Entwicklung eines eigenständigen Kompetenzbereichs für die Informationsgesellschaft und eine zunehmende Relevanz des Themas an.

Aus der industriepolitischen Sichtweise auf die Informationsgesellschaft entwickelten sich erste internetpolitische Kompetenzen. Ihr gegenüber stand eine wettbewerbs- und infrastrukturpolitische Perspektive. Die damit zusammenhängenden Kontroversen, die innerhalb des BMWi sowie zwischen dem BMWi und dem damaligen Bundesministerium für Post und Telekommunikation (BMPT) geführt wurden, bilden den Kontext der genannten Einschreibungen in die Organisationsstruktur. Inhaltlich bestanden Spannungen zwischen einem ökonomischen Diskurs, der Wettbewerb und Deregulierung stark betonte, und dem Diskurs zur Informationsgesellschaft, der eine eigene, ganzheitliche Strategie verlangte, z. B. eine Wirtschaftsförderung und Anpassungen von Datenschutz und Urheberrecht. Vertreter des rein ökonomischen Diskurses, unter anderem im bis 1998 bestehenden BMPT, betrachteten die Informationsgesellschaft eher als Fantasie und abstraktes Zukunftsthema (I6 2016). Dagegen wurden im BMWi reale Veränderungen der Wirtschaft durch die Informationstechnologien wahrgenommen und eine Strategie zur Begleitung dieser Umwälzungen als erforderlich angesehen (I3 2016; I6 2016). Der Konflikt wurde auch als Spannung zwischen einer wettbewerbs- und infrastrukturpolitischen Perspektive und einem anwendungsbezogenen Denken ,,vom Ende her“" wahrgenommen (I6 2016). Nicht zuletzt fühlten sich die Protagonisten des Diskurses um die Informationsgesellschaft im BMWi vom globalen Wettbewerb herausgefordert, und sie hielten daher industriepolitische Maßnahmen für notwendig, um den Anschluss nicht zu verpassen und die Versprechungen der Informationsgesellschaft realisieren zu können (I3 2016). Somit stand das Schutzgut „Wettbewerb“ dem Schutzgut „Informationsgesellschaft“ gegenüber, womit die IKT - inklusive Internet - schüt- 
zenswert wurden, um die künftige Dividende der Informationsgesellschaft nicht zu gefährden.

Diese Spannungen spiegelten sich auch in Diskussionen innerhalb des BMWi wider. Der damalige Wirtschaftsminister Günter Rexrodt nahm eine vermittelnde Rolle ein, insofern er beide Positionen für bedeutend hielt (I3 2016). Damit hatte der Diskurs zur Informationsgesellschaft Unterstützung durch einen Akteur mit politischer und administrativer Autorität.

Weitere Ereignisse außerhalb des BMWi waren jedoch ebenfalls bedeutend für die Gründung der Arbeitsgruppe „Informationsgesellschaft“" und deren Entwicklung zur Unterabteilung. Mitte der 1990er-Jahre beteiligte sich das BMWi an Expertengremien 7 . Vertreter aus Gesellschaft, Wirtschaft, Politik und öffentlicher Verwaltung erarbeiteten in ihnen ihre Expertise zum Thema „Informationsgesellschaft“. Die Gremien wurden auf Drängen der deutschen Industrie sowie auf Initiative des damaligen Forschungsministers Jürgen Rüttgers eingerichtet. Akteure, die für alternative Konzepte zur Informationsgesellschaft wie z. B. das der Kommunikationsgesellschaft (Tangens 1999) eintraten und die wirtschaftspolitische Einhegung des Diskurses der Informationsgesellschaft kritisierten ${ }^{8}$, waren nicht in den Gremien vertreten. Dazu gehörten NGOs wie der Chaos Computer Club (CCC) und das Forum InformatikerInnen für Frieden und gesellschaftliche Verantwortung (FIfF). Ebenso wenig vertreten waren Datenschützer.

Im Anschluss an diese Gremienarbeit folgte ein Kompetenzstreit mit dem Bundesministerium für Bildung und Forschung (BMBF) um die Federführung hinsichtlich des ersten internetrelevanten Gesetzes, dem sogenannten Multimedia-Gesetz ${ }^{9}$ von 1997 (BGBl. I, S. 1870), und hinsichtlich der Regierungsstrategie für die Informationsgesellschaft (BT-Drs. 13/4000; I3 2016). Diese Strategie gilt als ein wesentlicher Bezugspunkt für das Gesetz. Der Streit war auch ein Ringen darum, was für ein Thema die Informationsgesellschaft eigentlich ist: ein abstraktes Zukunftsthema, verbunden mit Forschung und Entwicklung, oder ein wirtschafts- und industriepolitisches Thema (I3 2016). Der Kompromiss zwischen den Häusern verschob die Federführung für das Gesetz in das BMBF und für die Strategieausarbeitung in das BMWi. Nach dem Regierungswechsel Ende 1998 wurden die Kompetenzen des BMBF dem BMWi übertragen. Die alleinig für die Informationsgesellschaft zuständige Unterabteilung „Informationsgesellschaft, Medienrecht“ entstand. Diese Zuständigkeitsverlagerung war eine Folge von Positionskämpfen im parteipolitischen Feld während der Koalitionsverhandlungen zwischen SPD und Grünen (I2 2015; I3 2016) und lediglich sekundär durch eine fachlich-inhaltliche Handlungsorientierung bedingt.

Die Thematisierung der Informationsgesellschaft durch internationale Institutionen (EU, G7) in der ersten Hälfte der 1990er-Jahre steht für die hohe Relevanz, die dem Diskurs dort beigemessen wurde. Der Diskurs diffundierte über den Bange-

\footnotetext{
7 Eines dieser Gremien war der sogenannte „Petersberg Kreis“. Ein anderes war der „Rat für Forschung, Technologie und Innovation“.

8 Akteure der Zivilgesellschaft sahen den Diskurs z. B. als „Revolution von oben“ (Bernhardt und Ruhmann 1996, S. 114).

9 Das Gesetz regelte u. a. das Providerprivileg und die Lizenzfreiheit von Internetunternehmen.
} 
mann-Bericht auf die nationale Ebene, wo er sich in der Arbeit von Expertengremien niederschlug und u. a. im Wirtschaftsminister Rexrodt einen Unterstützer fand. Auch deshalb ließen sich Deutungen, die mit dem Diskurs der Informationsgesellschaft verbunden waren, innerhalb des BMWi und gegenüber dem BMBF und BMPT nicht marginalisieren. Die Spannung zwischen den Deutungen außerhalb und innerhalb der Ministerien bildete den Kontext der Institutionalisierung des Diskurses. Die Informationsgesellschaft wurde im BMWi als industriepolitisches und gesellschaftspolitisches Thema verstanden, nicht bloß lediglich als Thema der Forschung oder Wettbewerbsregulierung. Das Thema bedürfe vielmehr einer eigenen Strategie. Ein Ministerialbeamter beschreibt die Zuständigkeit wie folgt:

Wir sind ja das Ministerium für Wirtschaft. Es besteht ein recht breiter Anspruch auf allgemeine Wettbewerbspolitik über Energiepolitik, Industriepolitik, Außenwirtschaftspolitik. Und ein neuer Strang, übergreifend als Konzept, war dann Informationsgesellschaft, als Sektor IT-Wirtschaft breiter gedacht, also wirtschaftlich gute gesellschaftliche Entwicklung. (I3 2016)

Das Thema „Informationsgesellschaft“ hat sich im Denken des Beamten zu einem abgrenzbaren Zuständigkeitsbereich - parallel zu anderen Bereichen wie z.B. dem der Energiepolitik - entwickelt, der seine materielle Entsprechung in der Unterabteilung „Informationsgesellschaft, Medienrecht“ mit alleiniger Zuständigkeit für die Informationsgesellschaft fand.

Mit der Entwicklung vom lokalen Experiment zur eigenen Unterabteilung begann im BMWi die Institutionalisierung der Internetpolitik als eigenständiger Kompetenzbereich. Über die Arbeit in Expertengremien, und unter weitgehendem Ausschluss von Diskurs-Kritikern, wurde der Diskurs der Informationsgesellschaft in das BMWi eingeschrieben und wirkte dort performativ. Das Ministerium war auf der Grundlage der in der ordentlichen Hierarchie verstetigten Arbeitsgruppe „Internetgesellschaft“ an der Einschreibung des Diskurses in eine Regierungsstrategie beteiligt, die als ein Bezugspunkt für das erste internetrelevante Gesetz in Deutschland gilt. Es positionierte sich somit in der entstehenden Internetpolitik, reproduzierte den Diskurs und trug dadurch zu dessen weiterer Institutionalisierung in der Breite bei. Jenseits von reinen wettbewerbs- oder forschungspolitischen Erwägungen betrieb das BMWi die Institutionalisierung eines Diskurses, in dem das Internet aus ökonomischem Kalkül als besonders und schützenswert galt, um die Versprechungen des Diskurses zu realisieren. Darin zeigen sich der heteronome Charakter der Positionierung sowie ein schwacher Autonomieanspruch.

\subsubsection{Institutionalisierung zwischen Digitalisierungs- und Innovationsdiskurs (seit 2014)}

In den 2000er-Jahren blieb die Zusammensetzung der Unterabteilung ,Informationsgesellschaft, Medienrecht“" relativ stabil (vgl. Online-Dokument 1). Erst ab 2014 fanden im Ministerium eine umfassende Reorganisation und auch Neuordnung von Zuständigkeiten statt, die in der Umbenennung der Abteilung VI „Innovations-, ITund Kommunikationspolitik“ in „Digital- und Innovationspolitik“ mündeten. Maßgeblich für diese Form der Institutionalisierung von Internetpolitik sind zum einen 
die Einschreibung des Diskurses zur Digitalisierung und zum anderen die Positionierung des Ministeriums im Innovationsdiskurs US-amerikanischer Prägung.

In technischer Hinsicht ist Digitalisierung die Überführung von analogen in digitale Signale. Seit ca. 2012 wird unter Digitalisierung aber ein tiefgreifender gesellschaftlicher Wandel verstanden, der, wie immer wieder betont wird, alle Lebensbereiche betrifft, wobei vor allem die Bereiche Wirtschaft und Arbeit hervorgehoben werden. Es ist das wirtschaftliche Potenzial, das vorwiegend mit der Digitalisierung assoziiert wird: die Hoffnung auf eine Steigerung von Effektivität, Effizienz und Wohlstandsgewinnen (Hofmann und Kniep 2018; König 2018, S. 402 ff.). Das Internet bzw. die digitale Vernetzung allgemein ist ein Bezugspunkt für diese wirtschaftlichen Hoffnungen und wird entsprechend gedeutet. Der Diskurs suggeriert jedoch auch, dass sich die Gesellschaft bereits inmitten der Digitalisierung befinde, der Prozess also unumkehrbar sei. Es gilt deshalb, die Chancen und Risiken $\mathrm{zu}$ antizipieren und sich neu zu erfinden, um in Zukunft bestehen zu können (Süssenguth 2015; BMWi 2017; I2 2015). Insofern weist der Diskurs durchaus einige Parallelen zum Diskurs der Informationsgesellschaft der 1990er-Jahre auf. „Digitalisierung“ - z. T. in Verbindung mit „Digitalpolitik“ - ist vor allem ein regierungsund wirtschaftsnaher Begriff in Abgrenzung zum eher oppositions- und zivilgesellschaftsnahen Begriff „Netzpolitik“, der zwischen 2006 und 2013 Konjunktur hatte (Hösl und Reiberg 2016; Hofmann und Kniep 2018). Unter beiden Begriffen wurden ähnliche Themen diskutiert. Es ist jedoch die wirtschaftliche Dimension, die Digitalisierung von Netzpolitik unterscheidet (Stemmer 2016, S. 11). Die Rede von Netzpolitik ist dagegen eher auf den Konflikt „Freiheit versus Sicherheit“ bezogen und hebt das freie und offene Internet in Verbindung mit Grund- und Bürgerrechten als Schutzgut hervor (Ganz 2018, S. 270; Sell 2017, S. 241 ff.).

Der Diskurs der Digitalisierung wird 2014 in die Abteilung „,Digital- und Innovationspolitik“ eingeschrieben, nachdem er 2014 in der Regierungsstrategie „Digitale Agenda“ verankert wurde (BMWi et al. 2014). Die Unterabteilung „Nationale und europäische Digitale Agenda“ befindet sich 2016 in der vierzügigen Abteilung „Digital- und Innovationspolitik“ (vgl. Online-Dokument 2). Sie besteht weitgehend aus den Zuständigkeiten für die Informationsgesellschaft aus den 1990er-Jahren. 2014 erhielt sie ein neues Referat mit Zuständigkeiten für „Ökonomische Fragen der Digitalen Agenda und gesellschaftliche Entwicklungen, Digitale Souveränität“" ${ }^{10}$

Die Änderung der Denomination von Abteilung VI hin zur „,Digitalpolitik“ geht auf einen ministeriumsinternen Vorschlag zu den Koalitionsverhandlungen zwischen CDU/CSU und SPD 2013 zurück (I3 2016). Mit dem Bezug auf das Digitale sollte ein umfassenderer Begriff genutzt und ,mehr zum [...] Paradigma“ gemacht werden, um dem sektorenübergreifenden Charakter der Digitalisierung gerecht zu werden (I3 2016). Die Entscheidung für „Digitalpolitik“ ist auch eine Entscheidung gegen „Netzpolitik“. Im Ministerium wurde „Digitalpolitik“ als neutral wahrgenommen, während „Netzpolitik“ mit der Zivilgesellschaft assoziiert wurde oder als ein zu enger Begriff empfunden wurde, weil er auf die (Netz-)Infrastruktur ziele (I2 2016; I6 2016).

10 Die Unterabteilung trat jedoch auch zwei Referate an die Unterabteilung „Ordnungsrahmen Digitalpolitik“ (ehemalige Kapazitäten des BMPT) ab und erhielt ein Referat aus diesem Bereich zurück. 
Der organisatorische Wandel durch das neue Referat „Ökonomische Fragen der Digitalen Agenda und gesellschaftliche Entwicklungen, Digitale Souveränität"11 steht im Kontext des hohen symbolischen Werts der Digitalisierung für die Regierung während der Koalitionsverhandlungen 2013 (I2 2015). Die Rolle des BMWi, als eines der drei hauptverantwortlichen Ministerien für die Digitale Agenda, war dabei ebenfalls ausschlaggebend. Für diese Wertschätzung der Digitalisierung ist aus Sicht der Ministerialbeamten die Bedrohung der nationalen Wettbewerbsfähigkeit verantwortlich. Vor diesem Hintergrund sollte zuvor vernachlässigten Themen wie z.B. der Sharing Economy und den digitalen Plattformen durch ein eigenes Referat mehr Gewicht gegeben werden (I3 2016). Es fand ein nachholender Aufbau von Kompetenzen und Expertise statt, der durch die Hausleitung unterstützt wurde.

Die öffentliche Positionierung des BMWi in diesen Zuständigkeitsbereichen erfolgte 2017 mit dem „Weißbuch Digitale Plattformen“ (BMWi 2017). Es wurde in einem Konsultationsverfahren erarbeitet, wodurch das BMWi die Expertise der Feldakteure konzentrierte und sich dadurch positionierte. Diese Publikation verdeutlichte die Relationalität der Positionierung mitunter durch die explizite Abgrenzung von der Idee der „Disruption“ und von den als marktbeherrschend wahrgenommenen Internetplattformen.

Das BMWi grenzte sich mit der Forderung „Transformation statt Disruption“ (ebd., S. $41 \mathrm{ff}$.) vom technikoptimistischen Innovationsdiskurs US-amerikanischer Internetunternehmen ab. Innovation und die Kreativität des Menschen gelten in diesem Diskurs als hohe Güter. Ihnen sollen keine (regulativen) Grenzen gesetzt werden, um mit disruptiven Technologien, die die bestehenden Institutionen aufbrechen, einen lebensbereichernden Fortschritt voranzutreiben (Dotson 2015). Allerdings macht sich das Ministerium zum Teil die Denkweise der Internetunternehmen selbst zu eigen, etwa wenn es temporäre „Experimentierräume“ ohne regulative Hürden vorschlägt (BMWi 2017, S. 79).

Das BMWi stellte sich jedoch nicht nur gegen die Disruption, die mit Unternehmen der Sharing Economy verbunden wird (z. B. Uber, AirBnB; I2 2015; I3 2016). Es positionierte sich auch gegen die dominante Stellung großer US-Internetunternehmen, indem es einen ,offenen, innovationsfördernden Wettbewerb“ im Internet statt ,inkompatible Insellösungen“ forderte (BMWi 2017, S. 57). Damit sind geschlossene Plattformen gemeint (z. B. Facebook), die durch ,Lock-in“-Praktiken Nutzer, Interaktionen und Daten vom Rest des Internets abzuschotten versuchen. Das Ministerium näherte sich durch diese Positionierung der Kritik der netzpolitischen Zivilgesellschaft an geschlossenen Plattformen an (Gerloff 2011) und so mittelbar deren Position eines Schutzes des offenen Internets.

Der Diskurs der Digitalisierung ähnelt dem Diskurs der Informationsgesellschaft der 1990er-Jahre hinsichtlich der Relevanz von ökonomischen Kategorien und der Betonung der Notwendigkeit des Wandels. Die Einschreibung des Diskurses in die Unterabteilung „Nationale und europäische Digitale Agenda“ und das Referat „Ökonomische Fragen der Digitalen Agenda und gesellschaftliche Entwicklungen, Digitale Souveränität“" stehen für eine Positionierung des BMWi, die gekennzeichnet ist

${ }^{11}$ Die Bezeichnung „Digitale Souveränität“ geht auf eine industriepolitische Positionierung in der Debatte um die Snowden-Enthüllungen von 2013 zurück (vgl. Pohle et al. 2016). 
vom Spannungsverhältnis von Autonomie und Heteronomie bzw. dem Spannungsverhältnis zwischen Internetpolitik und Wirtschaftspolitik. Das Ministerium nähert sich im Untersuchungszeitraum der Position „Schutz des offenen Internets“ an, jedoch aus ökonomischer Rationalität. Damit grenzt es sich von der Betonung von Netzfreiheit als Wert an sich und der Zivilgesellschaft ab, auch semantisch. Eine Einschreibung des Netzpolitik-Diskurses fand nicht statt bzw. lediglich marginal (vgl. BMWi 2017, S. 91). Trotz der Abgrenzung vom Konzept der Disruption übernimmt das Ministerium Teile des US-Innovationsdiskurses, der internetspezifische Geschäftsmodelle zu Schutzgütern erklärt. Auch hierin manifestiert sich eine heteronome Positionierung, jedoch unter Bezugnahme auf eine autonome Position, die etablierte nationale Institutionen (etwa Datenschutz oder Gewerbe) herausfordert.

\subsection{Bundesministerium des Innern: Von der IT-Sicherheit zur Cybersicherheit}

Auch das BMI hat sich im Diskurs zur Informationsgesellschaft positioniert - mit dem Programm „Innenpolitik für die Informationsgesellschaft“ aus dem Jahr 2000 (BT-Drs. 14/4055, S. 4), also etwas später als das BMWi. Zwar hinterließ auch diese Positionierung Spuren im Organigramm (vgl. Online-Dokument 3, Referat O 6, März 2000; I1 2015). Entscheidend für die Institutionalisierung von Internetpolitik im BMI waren jedoch IT-Sicherheitsdiskurse, die sich ab der Jahrtausendwende in der Aufbauorganisation des BMI materialisierten. Die dadurch entstandene Konfliktlinie zwischen IT-Sicherheitspolitik und etablierter Sicherheitspolitik verstetigte und verstärkte sich mit dem Cybersicherheitsdiskurs ab 2009, was sich Mitte 2014 in einer Neuorganisation der Aufbauorganisation niederschlug.

\subsubsection{Entstehung internetpolitischer Kompetenzen: IT-Sicherheitsdiskurse (1999-2002)}

In nationalen und internationalen Diskursen entstanden Ende der 1990er-Jahre teils apokalyptische Katastrophenszenarien im Zusammenhang mit dem sogenannten Jahr-2000-Problem bzw. dem Y2K-Bug (Eriksson 2001; Tapia 2003). Weil die Jahreszahl in etlichen Computerprogrammen lediglich durch die letzten beiden Ziffern codiert wurde, erwarteten zunächst einzelne IT-Spezialisten, dass die Datumsumstellung von 99 auf 00 bei der Jahrtausendwende zu massenhaften Systemfehlern führen würde. Beim transnationalen Transfer dieser computertechnischen Problemwahrnehmung in andere soziale Felder, etwa über Behörden in die Politik oder vermittelt über Massenmedien in die Öffentlichkeit, wurde das Y2K-Problem zunehmend sicherheitspolitisch, teils aber auch kulturell oder religiös aufgeladen (vgl. Tapia 2003). In das BMI gelangte die Y2K-Debatte Anfang 1999 durch IT-Experten des Bundesamts für Sicherheit in der Informationstechnik (BSI). Vor dem Hintergrund eines „Grundgefühls“ im Ministerium, dass IT-Themen wichtiger werden (I1 2015), wurden die Bedrohungsszenarien antizipiert und schufen ein Bewusstsein dafür, wie abhängig die eigene Handlungsfähigkeit von IT-Netzen war. Gleichzeitig wurden die IT-Netze durch die Bedrohungsszenarien als schützenswerte Güter entdeckt. Im Gegensatz zum Innovations- und Fortschrittsglauben des Diskurses zur Informationsgesellschaft manifestierten sich im IT-Sicherheitsdiskurs kurz vor der 
Jahrtausendwende die Ängste vor dem technologischen Wandel durch Computer und ihre Vernetzung. Die Ministerialbeamten befürchteten Probleme bei der Rentenauszahlung oder bei Grenzkontrollen, sollte die Polizeidatenbank „Inpol“ ausfallen. Das BMI bereitete den Jahreswechsel entsprechend vor: mit einer Notstandsverordnung und einem temporären Krisenstab, der die Silvesternacht im Ministerium verbrachte. Zwar blieben die befürchteten technischen Folgen des Jahrtausendwechsels weitgehend aus. Die Bedrohungswahrnehmung in Bezug auf IT und Internet blieb jedoch bestehen, auch aufgrund eines weiteren IT-Sicherheitsereignisses: das Computervirus „I love you“ (2000), das sich, verpackt im Mailanhang, über den Zugriff auf die Adresslisten von Internetnutzern verbreitete und internationale Aufmerksamkeit erlangte. Hinzu kamen im BMI Probleme mit der damals neuen Version der Polizeidatenbank ,Inpol“.

Vor diesem Hintergrund fiel 2001 die Entscheidung durch den damaligen Innenminister Otto Schily, die Kompetenzen für IT-Sicherheit im BMI neu zu organisieren (I1 2015; I5 2015). Im März 2002 wurde der IT-Stab gegründet, in dem bestehende Kompetenzen gebündelt wurden. Betroffen waren mindestens zwei Abteilungen, aus denen für den IT-Stab Kompetenzen abgezogen wurden: die Abteilung O ,Verwaltungsorganisation“ und die Abteilung IS „Innere Sicherheit“ (vgl. Online-Dokument 3). Die Einrichtung eines Stabes statt einer Abteilung sollte den internen Widerstand verringern. Wie Strukturneuerungen im Allgemeinen, stellte aber auch der IT-Stab für andere Abteilungen einen „Affront“ dar. Wenn Abteilungen Kompetenzen für neue Organisationseinheiten abgeben sollen, sei das eine „Kriegserklärung an den Apparat schlechthin. Institutionell empfindet man das so" (I1 2015). Der IT-Stab war organisationsübergreifend auf der Ebene des Staatssekretärs angesiedelt, was zusätzlich dazu beitrug, dass der Stab ,als quasi neues Politikfeld kritisch gesehen“ wurde (ebd.). Dass Ressourcen und Zuständigkeiten in Ministerien durch bestehende Akteure derart umkämpft sind, verdeutlicht, dass eine Politikfeldentstehung stets mit Widerständen etablierter Akteure verbunden ist.

Charakteristisch für die Politikfeldentstehung durch den IT-Stab war, erstens, der Übergang von einer reaktiven Diskursinstitutionalisierung in Bezug auf einzelne Problemwahrnehmungen (Y2K, Inpol) zu einer aktiven und zunehmend strategischen Positionierung in Bezug auf das Schutzgut IT. Anstatt den Stab nach der ausgebliebenen Y2K-Katastrophe sowie der Erneuerung von „Inpol“ wieder aufzulösen, erfolgte ein weiterer Kompetenzaufbau unter einem explizit programmatischen und strategischen Anspruch. Es entstanden in den frühen 2000er-Jahren drei angegliederte Projektgruppen: „KRITIS“ (Kritische IT-Infrastrukturen), ,Sicheres Internet“ und „Biometrie“, die auch als ,9/11-Folgen“ interpretiert werden (I1 2015). Die Projektgruppe „Biometrie“, zuständig für Pässe, Personalausweise und Meldewesen, verstetigte sich 2005 zum vierten Referat des IT-Stabs, der damit einen Abteilungsstatus erhielt. In dem Referat wurden technische Experten mit Umsetzungsverantwortlichen zusammengeführt, die daraufhin Gesetze entwarfen und Projekte durchführten (ebd.). Mit dem Biometrie-Referat wurde ein durch den Innenminister und internationale Diskurse getriebenes Thema institutionalisiert, das bis heute, vermittelt über die Überwachungsdebatten, Teil des internetpolitischen Diskurses ist.

Zweitens entstand mit der Gründung des Stabs eine thematische Eigenständigkeit der IT. Die Auswahl der Einheiten für den Stab orientierte sich an der Frage: ,Was 
sind denn ausschließliche IT-Referate?““(I5 2015). Die Eigenständigkeit bildete sich dabei in Abgrenzung zur Abteilung ÖS „Öffentliche Sicherheit“ heraus, d.h. relational zur etablierten Sicherheitspolitik. IT-relevante Referate mit direktem Bezug zu den Sicherheitsbehörden wurden nicht eingegliedert: „Wir wollten das außerhalb des Sicherheitsbereichs machen, mit Schwerpunkt Prävention und Schutz“ (ebd.). In der Abgrenzung reiner IT-Themen von der IT der Sicherheitsbehörden begann sich eine der zentralen politischen Konfliktlinien zwischen Internet- und Sicherheitspolitik zu institutionalisieren: der Konflikt zwischen dem Schutz informationstechnischer Systeme einerseits und dem Schutz öffentlicher Sicherheit durch IT-Kompetenzen von Sicherheitsbehörden andererseits. Dabei steht die Deutung von IT-Systemen als schützenswert der Deutung von IT-Systemen als Sicherheitsproblem gegenüber.

Internationale IT-Sicherheitsdiskurse mit Ereignischarakter, die auf medialer und fachinterner Ebene verhandelt wurden, haben sich in Form des IT-Stabes in der Aufbauorganisation des BMI materialisiert. Gerade der Y2K-Bug verdeutlicht die Folgen dominanter Diskurse. Eine „Technokalypse“ (Stevens 2016) blieb zwar aus; der Diskurs produzierte dennoch kognitive und materielle Effekte, unter anderem in der Organisation des BMI, die die Entwicklung von Internetpolitik dauerhaft prägten. Die Gründung des IT-Stabs und entsprechender Projektgruppen als Einschreibung internetbezogener Sicherheitsdiskurse illustriert die Responsivität der Ministerien gegenüber gesellschaftlichen Diskursen. Die darauf folgende programmatische Weiterentwicklung des Stabes verdeutlicht die performative Wirkung der Strukturen sowie der Positionierung des BMI als Akteur mit IT-Expertise. Unter dem Schutzgut IT-Sicherheit erfolgt eine Positionierung im internetpolitischen Feld, die sich im Spannungsverhältnis von Heteronomie und Autonomie interpretieren lässt. Relational zu Akteuren, die den Schutz des Internets und dessen eigener Regeln und Praktiken betonten (vgl. Sell 2017 zum Netzfreiheits-Diskurs), formierte sich mit der IT-Abteilung (Schutz des Internets als Faktor staatlicher Sicherheit) eine heteronome Position der Internetpolitik. Gleichzeitig steht die IT-Abteilung für eine schwache internetpolitische Autonomie gegenüber der Abteilung „Öffentliche Sicherheit" im BMI, in der die IT und das Internet nicht als schützenswert, sondern als Herausforderungen für etablierte Schutzgüter der Innenpolitik (etwa Kompetenzen zur Strafverfolgung) begriffen werden.

\subsubsection{Verschärfung eines Feld-Konflikts im Cybersicherheitsdiskurs (2009-2014)}

Bevor auf die Verschärfung des beschriebenen Konflikts im Cybersicherheitsdiskurs eingegangen wird, ist in diesem Fall relevant, welcher Diskurs sich nicht dauerhaft im BMI institutionalisiert hat: der Netzpolitik-Diskurs, der sich als Diskurs des autonomen Pols der Internetpolitik verstehen lässt. Anders als das BMWi positionierte sich das BMI zunächst aktiv und strategisch im Netzpolitik-Diskurs, als dieser im Zusammenhang mit der Piratenpartei und der Netzbewegung eine Hochphase hatte. Galt der Innenminister Wolfgang Schäuble unter dem Schlagwort „Stasi 2.0“ noch als klassischer Gegenspieler der Netzbewegung, setzte sein Nachfolger Thomas de Maizière ab 2009 auf einen dialogorientierten und dezidiert ,netzpolitischen“ Kurs 
(I8 2016). Der Anspruch von de Maizières 14 netzpolitischen Thesen ${ }^{12}$ sei gewesen, „einen Ordnungsrahmen für das Internet zu schaffen“ (I2 2015). Aufbauorganisatorisch schlug sich diese Positionierung im Jahr 2010 in Form der abteilungsübergreifenden „Projektgruppe Netzpolitik“ nieder. Der Anspruch der Projektgruppe war, ganzheitliche Grundsätze für Netzpolitik zu entwickeln und alle Abteilungen ,darauf einzuschwören“ (I1 2015). Dies gelang jedoch nicht dauerhaft. Die Projektgruppe wurde 2011 wieder aufgelöst, und der Begriff „Netzpolitik“ verschwand 2014 aus dem BMI. Es fand keine Fortschreibung des Netzpolitik-Diskurses statt, sondern ein semantisch-programmatischer Wandel durch das Aufgreifen des Digitalisierungsund vor allem des Cyberdiskurses.

Der Begriff „Cyber“ geht auf das wissenschaftliche Feld der Kybernetik der frühen 1950er-Jahre zurück (Rid 2016). In Deutschland, aber auch in den USA und anderen Ländern, hat sich der Begriff mittlerweile der kulturellen („Cyberpunk“) und internetutopischen („Cyberspace“) Konnotationen entledigt und sich in der ITund Sicherheitspolitik etabliert. In Deutschland, erklärte ein Interviewpartner, sei der Begriff „Cyber“ für vernetzte Bedrohungen etwa seit 2009 „Gesetz geworden“ (I7 2016). Helen Nissenbaum (2005, S. 65) interpretiert den semantischen Wandel von IT-Sicherheit zu Cybersicherheit in den USA als Verknüpfung eines technischen Diskurses von IT-Experten mit politischen Diskursen im Feld der nationalen Sicherheit. Der Blick auf die Diskursinstitutionalisierung im BMI als Teil der Politikfeldentstehung lässt jedoch die Interpretation $\mathrm{zu}$, dass sich im Cybersicherheitsdiskurs zwei politische Diskurse mit unterschiedlichen Schutzgütern teils überschneiden und teils kontrovers gegenüberstehen: ein internetpolitischer IT-Diskurs, der den Schutz technischer Infrastrukturen vor äußeren Eingriffen in den Mittelpunkt stellt, und ein sicherheitspolitischer Diskurs, in dem die staatliche Handlungsfähigkeit und öffentliche Sicherheit als schützenswert(er) erachtet werden und der die Eingriffspotenziale staatlicher Akteure in die IT legitimiert. Im BMI manifestiert sich diese Kontroverse im Konflikt zwischen der Abteilung IT und der Abteilung „Öffentliche Sicherheit“:

Diese ganzen Krypto-Debatten - da gibt es viele von - spielen sich im Innenministerium immer, aufbauorganisatorisch betrachtet, zwischen den Abteilungen IT und ÖS ab. Das heißt, seit zehn Jahren führen wir diese Debatten mit unterschiedlichen Positionen innerhalb des Ministeriums und tragen sie am Ende dem jeweiligen Minister vor. (I1 2015)

Es sei eine ständige Abwägungssache, wie weit einerseits nach rechtsstaatlichen Kriterien in die Kommunikation eingegriffen werden könne und inwieweit andererseits die Vertraulichkeit von Informationen gewährleistet werden muss (I4 2015). Dies wird von einem Ministerialbeamten auch als Spannungsverhältnis zwischen einer ,,präventiven“ und einer ,repressiven Komponente“ von Cybersicherheitspolitik interpretiert (I1 2015).

Vor dem Hintergrund der Enthüllungen von Edward Snowden über die Überwachungsprogramme der amerikanischen National Security Agency (NSA) und ihrer Partnerdienste im Jahr 2013 gewannen beide Seiten bzw. Diskurse an Bedeutung,

12 Die Thesen markierten den Abschluss der Dialogveranstaltungsreihe des BMI „Perspektiven deutscher Netzpolitik“. 
wodurch sich ihr kontroverses Verhältnis verschärfte. Einerseits wurden Schutzansprüche der IT vor staatlicher Überwachung begründbarer, auch im Hinblick auf den Schutz staatlicher Institutionen. Andererseits setzte die Snowden-Veröffentlichung neue Standards für staatliche Überwachungskompetenzen, an die sich die Sicherheitsbehörden anzupassen versuchen. Neben den Snowden-Enthüllungen prägten auch Terrorismusdebatten, insbesondere die Internetnutzung von Terroristen, den Cybersicherheitsdiskurs. Wenn die Internetkommunikation in das Zentrum sicherheitspolitischer Diskurse rückt, werden auch staatliche Eingriffe in die IT und das Internet begründbarer.

Im BMI lässt sich 2014 die Verschärfung der Kontroverse an den organisationalen Neuerungen im Rahmen der Digitalen Agenda ablesen. Die Neuorganisation entspricht einer Verstärkung beider Komponenten von Cybersicherheit - der IT- und der öffentlichen Sicherheit - durch den Aufbau von Ressourcen und eine stärkere Sichtbarkeit des Themas. Die präventive IT-Sicherheit wurde durch einen eigenen Stab (Stab IT II) ausgebaut, der den Schutz der Netze und der Internetnutzer zu gewährleisten beansprucht. Das Ergebnis ist eine Zweiteilung der Abteilung IT, die den neuen Namen „Informationstechnik, Digitale Gesellschaft und Cybersicherheit; IT-Direktor" trägt, in eine Unterabteilung und einen Stab (vgl. Online-Dokument 3, Juli 2014; I1 2015; I2 2015). Bemerkenswert ist das Referat ,Schutz der Bürgerinnen und Bürger im Internet; Datensicherheit“ (I3 2016). Dieser explizit programmatische Anspruch und die Bündelung verschiedener Aktivitäten unter diesem neuen Label sowie zusätzliche Ressourcen führten dazu, dass nun mit konkreten Maßnahmen „stärker Politik gemacht“ werde unter der Leitfrage: „Was können wir eigentlich zum Schutz der Bürger im Netz besser machen, um unsere Rolle auf dem Feld umzusetzen?“ (I1 2015). Die Abteilung IT ist die zweitgrößte Fachabteilung im BMI - nach der Abteilung „Öffentliche Sicherheit“ (ÖS). Auch dort wurden mit einer neuen Unterabteilung (Stab ÖS III) im Bereich der Bekämpfung von Kriminalität im Internet und der Cyberfähigkeiten von Sicherheitsbehörden Ressourcen aufgebaut (vgl. Online-Dokument 4).

Der Konflikthaftigkeit des Cybersicherheitsdiskurses war man sich bei der Neuorganisation bewusst, denn sie war das Produkt monatelanger interner Diskussionen. Ein Ergebnis war die Einrichtung einer Personalunion bei der Leitung der neuen Einheiten in den beiden Abteilungen. Der Stab „IT- und Cybersicherheit; sichere Informationstechnik“ (Stab IT II) der Abteilung IT und der Stab „Cybersicherheit im Bereich der Polizeien und des Verfassungsschutzes“ (Stab ÖS III) der Abteilung ÖS wurden von derselben Person geleitet. Dadurch entstand eine institutionelle Brücke zwischen der IT- und Cybersicherheit als Schutz von IT-Systemen einerseits und der Cybersicherheit als Kriminalitätsbekämpfung und IT-Kompetenz der Sicherheitsbehörden andererseits (vgl. Online-Dokument 4). Der Bedarf, im BMI zwischen beiden Seiten zu vermitteln, stieg mit der Verschärfung des Konflikts und manifestiert sich in der Personalunion, einer für Ministerien ungewöhnlichen Konstruktion, die mit den typischen Prinzipien der Linienorganisation bricht. Aus Sicht eines BMI-Beamten gehe es dabei um Koordination, aber auch um Außenwirkung und Interessenausgleich zwischen den Verfechtern unterschiedlicher Schutzgüter: Innenpolitikern und Sicherheitsbehörden auf der einen und Netzpolitikern und Datenschützern auf der anderen Seite (I1 2015). 
Die Entwicklung des Themas „Cybersicherheit“ im BMI zeigt, wie sich eine Kontroverse zwischen Internet- und Sicherheitspolitik im institutionellen Gefüge verstetigt und verschärft hat. Im Sinne einer wechselseitigen Verstetigung kognitiver Kategorien und materieller Strukturen findet so eine Institutionalisierung von Internetpolitik als IT- und Cybersicherheitspolitik in Abgrenzung zur etablierten Sicherheitspolitik und ihrem Verständnis von Cybersicherheit statt. Im BMI hat sich ein Feldkonflikt zwischen Autonomie und Heteronomie und zwischen Schutzgütern institutionalisiert, der auch durch Kontroversen mit anderen relevanten Akteuren im Feld (re)produziert wird. Das zeigt sich z. B. in Debatten zum Staatstrojaner, einer vom Bundeskriminalamt (BKA) eingesetzten Überwachungssoftware (Meister 2015). Eine spezifische Konstellation staatlicher Akteure (BMI, BSI, BKA) und nicht-staatlicher Akteure (Netzpolitik.org, CCC) mit bestimmten Problemwahrnehmungen ringt dabei um heteronomere oder autonomere Deutungen in der Auseinandersetzung um den Umgang mit Internetkommunikation.

\section{Zusammenfassung und Fazit}

Der Beitrag ging der Entstehung von Internetpolitik in Deutschland nach, verstanden als Prozess einer zunehmenden, aber durchaus konflikthaften und im ständigen Wandel begriffenen diskursiven Institutionalisierung eines neuen Politikfeldes. Im Fokus standen dabei zwei Ministerien, das BMI und das BMWi, die wir als zentrale Akteure in Politikfeldern verstehen, neben vielen weiteren Akteuren und Institutionen des politischen Feldes selbst (Parteien, Parlament, Regierung, Behörden) und aus anderen sozialen Feldern (Wirtschaft, Zivilgesellschaft).

Das konzeptionelle Repertoire von Feldtheorie und diskursivem Institutionalismus diente dazu, die mit dem Internet und der Digitalisierung einhergehenden gesellschaftlichen Transformationsprozesse nicht nur als Transformation bereits bestehender Felder, sondern als Entstehung eines neuen Politikfeldes zu interpretieren, in dem das Internet und die Digitalisierung zu einem gemeinsamen und umstrittenen Bezugspunkt einer bestimmten Konstellation von Akteuren werden, die sich in diesem Feld engagieren. Diesem Herangehen liegt eine Politikfeld-Konzeption zugrunde, die davon ausgeht, dass es nicht technikdeterministische, sondern gestaltende und interpretierende Praktiken und Prozesse sind, die das Internet und die Digitalisierung als ein neues Politikfeld konstituieren. Anders als bei einer neo-institutionalistischen Perspektive auf die Entstehung internetpolitischer Zuständigkeiten lag deshalb der Fokus der Untersuchung auf den Deutungskonflikten und -unterschieden zwischen den Akteuren und damit auf einer „Logik der Abgrenzung“ statt auf institutionellen Anpassungsprozessen nach einer „Logik der Angemessenheit“.

Das Ziel der Untersuchung war es, einen Beitrag zur konzeptionellen Fundierung von Politikfeldern und ihrer Entstehung am Beispiel der Internetpolitik zu leisten. Politikfelder sind relativ autonome Produktionsräume für politische Kategorien und Handlungsoptionen, in deren Zentrum als gemeinwohlrelevant anerkannte Schutzgüter stehen. Politikfelder entstehen also, wenn neue Schutzgüter auftauchen bzw. entdeckt werden und sich im Zuge einer wechselseitigen Verstärkung von semantischen und institutionellen Neuerungen etablieren. Wie z. B. auch im Fall der 
europäischen Inklusionspolitik (Bernhard 2010), lässt sich für die Internetpolitik ein semantischer Wandel der dominanten Streitgegenstände und Schutzgüter feststellen. Dieser Wandel vollzog sich in den 1990er- und 2000er-Jahren von der ,Informationsgesellschaft“ über die „Netzpolitik“ hin zur „Digital- und Cyberpolitik“. Er lässt sich auch im Hinblick auf das Spannungsverhältnis von Heteronomie und Autonomie interpretieren. Jene Diskurse, die die Besonderheit, das Neue und die übergeordnete Bedeutung der Schutzgüter eines Politikfeldes hervorheben, lassen sich als autonome Positionen oder Diskurse verstehen. Sie stehen mit heteronomen Diskursen etablierter Politikfelder in Konflikt, die an bereits bestehende eigene Schutzgüter anknüpfen und neu auftauchende Themen diesen weitgehend unterordnen. Bei der Internetpolitik handelt es sich nur bedingt um eine Geschichte der zunehmenden Autonomisierung eines Politikfeldes. Zwar nimmt die Autonomie der entsprechenden Abteilungen in den beiden hier untersuchten Ministerien zu, wodurch auch die Eigenständigkeit des Feldes institutionalisiert wird. Allerdings werden die gesellschaftlich virulenten Netzpolitik-Diskurse des autonomen Pols nicht übernommen, zugunsten eigener, eher heteronomer Konzepte wie „Digitalpolitik“ und „Cyber“. In dieser Hinsicht lassen sich nicht nur Parallelen zur Inklusionspolitik ziehen, sondern auch zur Entstehung von Umweltpolitik. Auch die Umweltpolitik entstand 1969 zunächst innerhalb des Innenministeriums (Müller 1986, S. 72). Im Unterschied zur Umweltpolitik ist es bei der Internetpolitik jedoch noch offen, ob es zu einer weiteren Autonomisierung des Feldes kommt oder ob sie ein Politikfeld mit schwacher Autonomie bleibt.

Der Beitrag hat nachgezeichnet, wie sich die Internetpolitik auf der Ebene zweier Ministerien institutionalisiert hat. In der bisherigen Internetpolitik-Forschung wurde die Ministerialverwaltung vernachlässigt. Sowohl das BMI als auch das BMWi haben sich mit dem Aufbau von eigenen Abteilungen im internetpolitischen Feld positioniert. Die Ministerien treten dadurch als Akteure in jeweils zwei, teilweise miteinander konkurrierenden Politikfeldern auf: der Internetpolitik und der Sicherheitsbzw. Wirtschaftspolitik. Der semantische Wandel in den Organigrammen zeigt, dass in den beiden untersuchten Ministerien zu verschiedenen Zeitpunkten unterschiedliche, wenngleich nicht voneinander unabhängige Diskurse die Entstehung von Internetpolitik vorantrieben. Die ab 2014 an der Digitalen Agenda der Bundesregierung ausgerichteten Neueinschreibungen weisen wiederum eine stärker ministerienübergreifende Semantik auf. Der digitalpolitische Diskurs vermochte somit eine stärkere semantische Brücke herzustellen als der Diskurs der Informationsgesellschaft in den 1990er-Jahren. Doch auch die mit der Digitalen Agenda verknüpften veränderten Denominationen von Abteilungen und Referaten sind grundsätzlich kontingent. Der Begriff der Netzpolitik, der etwa ab 2006 auf der Ebene der Parteien, Zivilgesellschaft und Medien Konjunktur hatte, wurde ihnen nicht eingeschrieben. Im BMI, das im Vergleich zum BMWi stärker im Fokus der Debatten um Netzsperren und Überwachung stand, wurde der Netzpolitik-Diskurs nur vorübergehend aufgegriffen. Der Begriff galt als zu sehr mit einer spezifischen Community - den Internetaktivisten und der Piratenpartei - verbunden oder als zu eng, um alle digitalpolitisch relevanten Themen abzudecken. Der Begriff der Digitalisierung und die daraus abgeleitete Rede von Digitalpolitik wurden als übergreifender, breiter und neutraler 
empfunden. Doch gerade die Nutzung eines scheinbar neutralen Gegenbegriffs zu Netzpolitik macht dessen politische Dimension sichtbar.

In aufbauorganisatorischer Hinsicht sind sowohl im BMWi als auch im BMI die ersten internetrelevanten Kompetenzen in Organisationseinheiten entstanden, die außerhalb der ordentlichen Hierarchie angesiedelt waren, wie Arbeitsgruppen, Projektgruppen oder Stäbe. Bis zum Jahr 2016 haben sich in beiden Ministerien aus den lokalen Experimenten außerhalb der Linienorganisation eigenständige Abteilungen innerhalb der ordentlichen Hierarchie entwickelt. Trotz der Einordnung der Internetpolitik in bestehende Ministerien etablierte sie sich als ein eigenständiges Thema, das sich bereits 1995 (BMWi) und 2002 (BMI) einer vollständigen Einbettung in bereits existierende Verantwortlichkeiten und Organisationsstrukturen entzog. Dadurch entstanden relativ autonome Einheiten mit eigenen, auf das Internet bezogenen Schutzgütern: der Schutz von IT-Netzen (im Fall des BMI) und der Schutz einer spezifischen Internetwirtschaft (im Fall des BMWi). Diese Schutzgüter wurden durch eigene Abteilungen institutionalisiert und gehen mit Autonomieansprüchen gegenüber der etablierten Wirtschafts- und Sicherheitspolitik einher. Dass es bislang kein eigenständiges Digital- oder Internetministerium gibt, sollte also nicht darüber hinwegtäuschen, dass es innerhalb der Ministerien internetpolitische Abteilungen mit einem gewissen Autonomieanspruch gibt. Die beiden Ministerien sind deshalb weder nur die sicherheits- oder wirtschaftspolitischen Gegenspieler einer kleinen „Netzgemeinde“, noch ist die Internetpolitik für das politische Feld völliges „Neuland“, wie es 2013 in einer Rede von Angela Merkel hieß. Anders als manche Studien zur Internetpolitik nahelegen (wie etwa die von Scheffel 2016), reicht die Entstehung von Internetpolitik weiter zurück als bis zum Beginn der öffentlichkeitswirksamen Phase der Netzpolitik ab 2009.

Gleichzeitig bleibt festzuhalten, dass die Autonomie der Internetpolitik in den Ministerien stets durch die bereits etablierten Schutzgüter von Wirtschafts- und Sicherheitspolitik eingehegt blieb und durch sie bis heute herausgefordert wird, also heteronomen Einflüssen unterliegt. Das zeigen im BMI die Konflikte und Kooperationen zwischen den Abteilungen IT und ÖS. Im BMWi manifestieren sich die heteronomen Einflüsse im Eintreten für einen Schutz des Internets aus ökonomischer Rationalität sowie einem Schutz etablierter Gewerbe und Geschäftsmodelle. Insgesamt werden die heteronomen Einflüsse in beiden Ministerien auch dadurch sichtbar, dass der auf eine starke Autonomie zielende zivilgesellschaftliche Diskurs der Netzpolitik, der das freie und offene Internet als schützenswertes Gut definiert, nicht institutionalisiert wurde. Bei der staatlichen Internetpolitik in Deutschland handelt es sich bislang um ein schwach autonomes Politikfeld.

Die hier vorgestellte Untersuchung zur Institutionalisierung des Feldes der Internetpolitik unterliegt auch einigen Limitierungen, unter anderem hinsichtlich der Umsetzung des feldtheoretischen Ansatzes. Zum einen wurden nur die symbolischen, nicht die objektiven Relationen der Feldakteure untersucht. Die symbolischen Relationen wurden mittels des Ansatzes des diskursiven Institutionalismus weiter ausbuchstabiert. Jedoch blieben die „Kapitalausstattungen“ der Akteure unbeleuchtet, abgesehen von der vorausgesetzten Ressourcen-Konzentration in den Abteilungen. Zum anderen konnten durch den Fokus auf Ministerien als zentrale Akteure nationaler Politikfelder nicht alle feldkonstituierenden Akteure und Elemente und 
deren Relationen betrachtet werden. Diverse internetpolitische Konflikte, die mitunter durchaus feldkonstituierend gewesen sein mögen, aber nicht unter Beteiligung der betrachteten Ministerien ausgetragen wurden, sind durch den gewählten Fokus zwangsläufig aus der Betrachtung herausgefallen. Dazu gehört z. B. etwa der Streit um Netz-Standards in den 1980er-Jahren (Werle 2000; Hofmann 2019). Dazu gehören aber auch die Dauerkonflikte zwischen diversen zivilgesellschaftlichen Akteuren und den IT- und Internet-Konzernen. Sie sollten Gegenstand einer umfassenderen Erzählung der deutschen Internetpolitik sein, die das Politikfeld in seiner Gänze beleuchtet.

Funding Open Access funding provided by Projekt DEAL.

Open Access Dieser Artikel wird unter der Creative Commons Namensnennung 4.0 International Lizenz veröffentlicht, welche die Nutzung, Vervielfältigung, Bearbeitung, Verbreitung und Wiedergabe in jeglichem Medium und Format erlaubt, sofern Sie den/die ursprünglichen Autor(en) und die Quelle ordnungsgemäß nennen, einen Link zur Creative Commons Lizenz beifügen und angeben, ob Änderungen vorgenommen wurden.

Die in diesem Artikel enthaltenen Bilder und sonstiges Drittmaterial unterliegen ebenfalls der genannten Creative Commons Lizenz, sofern sich aus der Abbildungslegende nichts anderes ergibt. Sofern das betreffende Material nicht unter der genannten Creative Commons Lizenz steht und die betreffende Handlung nicht nach gesetzlichen Vorschriften erlaubt ist, ist für die oben aufgeführten Weiterverwendungen des Materials die Einwilligung des jeweiligen Rechteinhabers einzuholen.

Weitere Details zur Lizenz entnehmen Sie bitte der Lizenzinformation auf http://creativecommons.org/ licenses/by/4.0/deed.de.

\section{Literatur}

Audenhove, L. v., Burgelman, J.-C., Cammaerts, B., \& Nulens, G. (2003). Discourse and reality in international information society policy. The dominant scenario and its application in the developing world. Communicatio, 29, 79-113.

Berger, P. L., \& Luckmann, T. (1966). The social construction of reality. A treatise in the sociology of knowledge. Garden City: Doubleday.

Bernhard, S. (2010). Die Konstruktion von Inklusion. Europäische Sozialpolitik aus soziologischer Perspektive. Frankfurt a. M.: Campus.

Bernhard, S. (2011). Beyond constructivism. The political sociology of an EU policy field. International Political Sociology, 5, 426-445.

Bernhardt, U., \& Ruhmann, I. (1996). Revolution von oben. Der Weg in die Informationsgesellschaft. In J. Tauss, J. Kollbeck, \& J. Mönikes (Hrsg.), Deutschlands Weg in die Informationsgesellschaft. Herausforderungen und Perspektiven für Wirtschaft, Wissenschaft, Recht und Politik (S. 114-129). Baden-Baden: Nomos.

BMWi (2017). Weißbuch Digitale Plattformen. Digitale Ordnungspolitik für Wachstum, Innovation, Wettbewerb und Teilhabe. https://www.bmwi.de/Redaktion/DE/Publikationen/Digitale-Welt/weissbuchdigitale-plattformen.pdf?_blob=publicationFile\&v=22. Zugegriffen: Mai 2017.

BMWi, BMI, \& BMVI (2014). Digitale Agenda. Ressortabgestimmter Entwurf. Stand: 28. Juli 2014. https://netzpolitik.org/2014/digitale-agenda-der-bundesregierung-wir-veroeffentlichen-denmittlerweile-abgestimmten-entwurf/. Zugegriffen: Aug. 2018.

Bourdieu, P. (1993). Sozialer Sinn. Kritik der theoretischen Vernunft. Frankfurt a. M.: Suhrkamp.

Bourdieu, P. (1994). Rethinking the state. Genesis and structure of the bureaucratic field. Sociological Theory, 12, 1-18.

Bourdieu, P. (1999). Die Regeln der Kunst. Genese und Struktur des literarischen Feldes. Frankfurt a. M.: Suhrkamp.

Clark, D. D. (2016). The contingent Internet. Daedalus, 145, 9-17.

Derlien, H.-U. (1995). Public administration in Germany: Political and societal relations. In J. Pierre (Hrsg.), Bureaucracy in the modern state. An introduction to comparative public administration (S. 64-91). Cheltenham: E. Elgar. 
Diaz-Bone, R. (2006). Die interpretative Analytik als methodologische Position. In B. Kerchner \& S. Schneider (Hrsg.), Foucault: Diskursanalyse der Politik. Eine Einführung (S. 68-84). Wiesbaden: VS Verlag für Sozialwissenschaften.

DiMaggio, P. J., \& Powell, W. W. (1983). The iron cage revisited. Institutional isomorphism and collective rationality in organizational fields. American Sociological Review, 48, 147-160.

Döhler, M. (2015). Das Politikfeld als analytische Kategorie. dms - der moderne staat. Zeitschrift für Public Policy, Recht und Management, 8, 51-69.

Dölemeyer, A., \& Rodatz, M. (2010). Diskurse und die Welt der Ameisen. Foucault mit Latour lesen (und umgekehrt). In R. Feustel \& M. Schochow (Hrsg.), Zwischen Sprachspiel und Methode. Perspektiven der Diskursanalyse (S. 197-220). Bielefeld: transcript.

Dotson, T. (2015). Technological determinism and permissionless innovation as technocratic governing mentalities. Psychocultural barriers to the democratization of technology. Engaging Science, Technology, and Society, 1, 98-120.

Dubois, V. (2015). The fields of public policy. In M. Hilgers \& E. Mangez (Hrsg.), Bourdieu's theory of social fields. Concepts and applications (S. 199-220). New York: Routledge.

Emirbayer, M., \& Johnson, V. (2008). Bourdieu and organizational analysis. Theory and Society, 37, 1-44.

Eriksson, J. (2001). Cyberplagues, IT and security: Threat politics in the information age. Journal of Contingencies and Crisis Management, 9, 200-210.

European Commission (1994): Growth, competitiveness and employment. White paper follow-up. Luxembourg: Office for Official Publications of the European Communities. http://aei.pitt.edu/1199/1/info_ society_bangeman_report.pdf. Zugegriffen: Juli 2016.

Fleischer, J., Bertels, J., \& Schulze-Gabrechten, L. (2018). Stabilität und Flexibilität. Wie und warum ändern sich Ministerien? Baden-Baden: Nomos.

Fligstein, N., \& Vandebroeck, D. (2014). The frenzy of fields: An interview with Neil Fligstein on field theory and social skill. Irish Journal of Sociology, 22, 107-129.

Ganz, K. (2018). Die Netzbewegung. Subjektpositionen im politischen Diskurs der digitalen Gesellschaft. Leverkusen: Verlag Barbara Budrich.

Geczy-Sparwasser, V. (2003). Die Gesetzgebungsgeschichte des Internet. Berlin: Duncker \& Humblot.

Gerloff, K. (2011). Das neue Facebook: Nix wie raus hier. netzpolitik.org. https://netzpolitik.org/2011/dasneue-facebook-nix-wie-raus-hier/. Zugegriffen: Mai 2018.

Gorski, P. S. (2013). Bourdieusian theory and historical analysis. Maps, mechanisms, and methods. In P. S. Gorski (Hrsg.), Bourdieu and historical analysis (S. 327-366). Durham: Duke University Press.

Guiraudon, V. (2003). The constitution of a European immigration policy domain: A political sociology approach. Journal of European Public Policy, 10, 263-282.

Hajer, M. A. (1995). The politics of environmental discourse. Ecological modernization and the policy process. Oxford: Clarendon Press.

Haunss, S., \& Hofmann, J. (2015). Entstehung von Politikfeldern - Bedingungen einer Anomalie. dms der moderne staat. Zeitschrift für Public Policy, Recht und Management, 8, 29-49.

Hofmann, J. (2019). Mediated democracy - Linking digital technology to political agency. Internet Policy Review, 8(2), 1-18.

Hofmann, J., \& Kniep, R. (2018): Die Pop-Karriere der deutschen Netzpolitik: Eine Erfolgsgeschichte? re:publica 18, 05.04.2018. https://18.re-publica.com/en/session/pop-karriere-deutschen-netzpolitikerfolgsgeschichte. Zugegriffen: Juli 2018.

Hösl, M., \& Reiberg, A. (2016). Netzpolitik in statu nascendi. Eine Annäherung an Wegmarken der Politikfeldgenese. In M. Lemke \& G. Wiedemann (Hrsg.), Text Mining in den Sozialwissenschaften. Grundlagen und Anwendungen zwischen qualitativer und quantitativer Diskursanalyse (S. 315-342). Wiesbaden: Springer VS.

Hösl, M., Irgmaier, F., \& Kniep, R. (2019). Diskurse der Digitalisierung und organisationaler Wandel in Ministerien. In T. Klenk, F. Nullmeier, \& G. Wewer (Hrsg.), Handbuch Digitalisierung in Staat und Verwaltung. Wiesbaden: Springer VS. https://doi.org/10.1007/978-3-658-23669-4_33-1.

Howlett, M., Ramesh, M., \& Perl, A. (2009). Studying public policy. Policy cycles and policy subsystems. Ontario: Oxford University Press Canada.

Hustedt, T. (2013). Ministerialverwaltung im Wandel. Struktur und Rolle der Leitungsbereiche im deutschdänischen Vergleich. Baden-Baden: Nomos.

Jørgensen, R. F. (2012). Framing the net. How discourse shapes law and culture. Ph.D. dissertation, Department of Communication, Business and Information Technologies. Kopenhagen: Roskilde University. 
Kirchner, S., Krüger, A. K., Meier, F., \& Meyer, U. (2015). Wie geht es weiter mit dem soziologischen Neo-Institutionalismus? Eine kritische Auseinandersetzung. In M. Apelt \& U. Wilkesmann (Hrsg.), Zur Zukunft der Organisationssoziologie (S. 189-202). Wiesbaden: Springer VS.

Knoke, D. (2004). The sociopolitical construction of national policy domains. In C. H. C. A. Henning \& C. Melbeck (Hrsg.), Interdisziplinäre Sozialforschung. Theorie und empirische Anwendungen (S. 81-96). Frankfurt a. M.: Campus.

König, P. D. (2018). Digitalpolitische Positionen im deutschen Parteiensystem. Eine Analyse der Parteipositionen zu den Bundestagswahlen der Jahre 2009, 2013 und 2017. Zeitschrift für Vergleichende Politikwissenschaft, 12, 399-427.

Kropf, J. (2015). Konsekrationsinstanzen im digitalen Wandel. Beiträge zur Erweiterung des feldanalytischen Forschungsprogramms am Beispiel populärer Musik. Berliner Journal für Soziologie, 25, 429-458.

Lang, A. (2007). Die Evolution sektoraler Wirtschaftsverbände: Informations- und Kommunikationsverbände in Deutschland, Großbritannien und Spanien. Wiesbaden: VS Verlag für Sozialwissenschaften.

Latour, B. (1999). Pandora's hope. Essays on the reality of science studies. Cambridge: Harvard University Press.

Lichtmannegger, C. (2018). Task environment matters for intra-ministerial change. The interaction of international environment, organizational and opportunity factors. International Journal of Public Administration, 87, 1-14.

Loer, K., Reiter, R., \& Töller, A. E. (2015). Was ist ein Politikfeld und warum entsteht es? dms - der moderne staat. Zeitschrift für Public Policy, Recht und Management, 8, 7-28.

Meister, A. (2015). Geheime Kommunikation: BSI programmierte und arbeitete aktiv am Staatstrojaner, streitet aber Zusammenarbeit ab. netzpolitik.org. https://netzpolitik.org/2015/geheimekommunikation-bsi-programmierte-und-arbeitete-aktiv-am-staatstrojaner-streitet-aberzusammenarbeit-ab/. Zugegriffen: Sept. 2017.

Mérand, F. (2010). Pierre Bourdieu and the birth of European defense. Security Studies, 19, 342-374.

Meyer, J. W., \& Rowan, B. (1977). Institutionalized organizations: Formal structure as myth and ceremony. American Journal of Sociology, 83, 340-363.

Müller, E. (1986). Innenwelt der Umweltpolitik. Sozial-liberale Umweltpolitik - (Ohn)Macht durch Organisation? Opladen: Westdeutscher Verlag.

Niedermayer, O. (2013). Die netzpolitischen Reaktionen der anderen Parteien auf das Erscheinen der Piratenpartei. In O. Niedermayer (Hrsg.), Die Piratenpartei (S. 237-257). Wiesbaden: Springer VS.

Nimmo, R. (2011). Actor-network theory and methodology: Social research in a more-than-human world. Methodological Innovations Online, 6, 108-119.

Nissenbaum, H. (2005). Where computer security meets national security. Ethics and Information Technology, 7, 61-73.

Phillips, N., Lawrence, T. B., \& Hardy, C. (2004). Discourse and institutions. The Academy of Management Review, 29, 635-652.

Pohle, J., Hösl, M., \& Kniep, R. (2016). Analysing internet policy as a field of struggle. The discursive institutionalisation of key conflicts in the German internet policy field. Internet Policy Review, 5, $1-21$.

Richter, P. (2012). Die Organisation öffentlicher Verwaltung. In M. Apelt \& V. Tacke (Hrsg.), Handbuch Organisationstypen (S. 91-112). Wiesbaden: Springer VS.

Rid, T. (2016). Maschinendämmerung. Eine kurze Geschichte der Kybernetik. Berlin: Propyläen.

Scheffel, F. (2016). Netzpolitik als Policy Subsystem? Internetregulierung in Deutschland 2005-2011. Baden-Baden: Nomos.

Schmidt-Wellenburg, C. (2013). Diskursiver Wandel im Fadenkreuz von Wissenssoziologischer Diskursanalyse und Feldanalyse. Der Aufstieg der Managementberatung. In R. Keller \& I. Truschkat (Hrsg.), Methodologie und Praxis der Wissenssoziologischen Diskursanalyse (S. 451-480). Wiesbaden: Springer VS.

Schwanholz, J. (2019). Der Deutsche Bundestag als Akteur in der Netzpolitik. In A. Busch, Y. Breidl \& T. Jakobi (Hrsg.), Netzpolitik. Ein einführender Überblick (S. 105-125). Wiesbaden: Springer VS.

Schwartz-Shea, P., \& Yanow, D. (2012). Interpretive research design. Concepts and processes. New York: Routledge.

Scott, W. R. (2014). Institutions and organizations. Ideas, interests, and identities. 4th edition. Los Angeles: Sage.

Sell, S. (2017). Kommunikationsfreiheit. Emanzipatorische Diskurse im Kontext medientechnologischer Entwicklungsprozesse. Wiesbaden: Springer VS. 
Sending, O. J. (2015). The politics of expertise. Competing for authority in global governance. Ann Arbor: University of Michigan Press.

Stemmer, M. (2016). Digitale Governance - ein Diskussionspapier. Berlin: Fraunhofer-Institut für Offene Kommunikationssysteme FOKUS. http://www.oeffentliche-it.de/documents/10181/14412/ Digitale+Governance+-+Ein+Diskussionspapier. Zugegriffen: Juni 2016.

Stevens, T. (2016). Cyber security and the politics of time. Cambridge: Cambridge University Press.

Süssenguth, F. (2015). Die Organisation des digitalen Wandels. Zur Funktion von Digitalisierungssemantiken in Wirtschaft, Medien und Politik. In F. Süssenguth (Hrsg.), Die Gesellschaft der Daten. Über die digitale Transformation der sozialen Ordnung (S. 93-122). Bielefeld: transcript.

Tangens, R. (1999). BürgerInnenvernetzung braucht Medienkompetenz. https://www.boell-hessen.de/ archivseite/pol/tangens.htm. Zugegriffen: Sept. 2017.

Tapia, A.H. (2003). Technomillennialism: A subcultural response to the technological threat of Y2K. Science, Technology and Human Values, 28, 483-512.

Tolbert, P. S., \& Zucker, L. G. (1999). The institutionalization of institutional theory. In S. R. Clegg \& C. Hardy (Hrsg.), Studying organization. Theory \& method (S. 169-184). London: Sage.

Weber, M. (1980). Wirtschaft und Gesellschaft. Grundriß der verstehenden Soziologie. 5., rev. Auflage. Tübingen: Mohr.

Wendelin, M., \& Löblich, M. (2013). Netzpolitik-Aktivismus in Deutschland. Deutungen, Erwartungen und Konstellationen zivilgesellschaftlicher Akteure in der Internetpolitik. Medien \& Kommunikationswissenschaft, 61, 58-75.

Werle, R. (2000). The impact of information networks on the structure of political systems. In C. Engel \& K. H. Keller (Hrsg.), Understanding the impact of global networks on local social, political and cultural values (S. 167-192). Baden-Baden: Nomos.

Maximilian Hösl geb. 1985. Wissenschaftlicher Mitarbeiter am Wissenschaftszentrum Berlin für Sozialforschung (WZB) in der Projektgruppe „Politik der Digitalisierung“. Forschungsschwerpunkte: Feldsoziologie, Institutionentheorie, Internetpolitik, Verwaltungsforschung. Ausgewählte Veröffentlichungen: (mit R. Kniep und F. Irgmeier) Digitalisierungsdiskurse und Ministerialorganisation, in: T. Klenk, F. Nullmeier \& G. Wewer (Hrsg.), Digitalisierung in Staat und Verwaltung, 2019; Semantics of the Internet: A political history, in: Internet Histories, 2019.

Ronja Kniep geb. 1987. Wissenschaftliche Mitarbeiterin am Wissenschaftszentrum Berlin für Sozialforschung (WZB) in der Projektgruppe „Politik der Digitalisierung“. Doktorandin an der Freien Universität Berlin. Forschungsschwerpunkte: Feldtheorie, Internetpolitik, Cybersicherheit, Kommunikationsüberwachung. Ausgewählte Veröffentlichungen: (mit J. Pohle und M. Hösl) Analysing internet policy as a field of struggle, in: Internet Policy Review, 2016; Media accountability online in Israel. An application of Bourdieu's field theory, in: Global Media Journal, 2015. 